CTP-SCU/2015013

\title{
Solutions in Bosonic String Field Theory and Higher Spin Algebras in $A d S$
}

\author{
Dimitri Polyakov ${ }^{\dagger(1),(2)}$ \\ Center for Theoretical Physics (1) \\ College of Physical Science and Technology \\ Sichuan University, Chengdu 610064, China \\ Institute for Information Transmission Problems $(\text { IITP })^{(2)}$ \\ Bolshoi Karetny per. 19/1 \\ 127994 Moscow, Russia
}

\begin{abstract}
We find a class of analytic solutions in open bosonic string field theory, parametrized by the chiral copy of higher spin algebra in $A d S_{3}$. The solutions are expressed in terms of the generating function for the products of Bell polynomials in derivatives of bosonic space-time coordinates $X^{m}(z)$ of the open string, which form is determined in this work. The products of these polynomials form a natural operator algebra realizations of $w_{\infty}$ (area-preserving diffeomorphisms), enveloping algebra of $\mathrm{SU}(2)$ and higher spin algebra in $A d S_{3}$. The class of SFT solutions found can, in turn, be interpreted as the "enveloping of enveloping", or the enveloping of $A d S_{3}$ higher spin algebra. We also discuss the extensions of this class of solutions to superstring theory and their relations to higher spin algebras in higher space-time dimensions.
\end{abstract}

July 2015

$\dagger(1),(2)$ polyakov@sogang.ac.kr ; polyakov@scu.edu.cn; twistorstring@gmail.com 


\section{Introduction}

It is well-known that the equations of motion of Witten's cubic string field theory [1], [2]:

$$
Q \Psi+\Psi \star \Psi=0
$$

resemble the Vasiliev's equations in the unfolding formalism in higher-spin theories [3]

$$
d W+W \wedge \star W=0
$$

(flatness condition for connection in infinite-dimensional higher-spin algebras) that determine the interactions of the higher-spin gauge fields in this formalism, along with equations for other master fields, containing higher-spin Weyl tensors and auxiliary fields (see also e.g. [4], [5], [6] for the works/reviews on this remarkable formalism). Higher spin holography strongly hints, however, that this resemblance may be much more than just a formal similarity. The generalized 1-form $W$ of (2) contains all the higher-spin gauge fields components in $A d S$ spaces which, by holography principle, are related to various multi-index composite operators in the dual CFT's. Any of these CFT's, in turn, must be a low-energy limit of string theory in $A d S_{d+1}$, with the $C F T_{d}$ correlators reproduced by the worldsheet correlation functions of the vertex operators in $A d S$ string theory, with the space-time fields polarized along the boundary of the $A d S$ space. On the other hand, the second-quantized string field $\Psi$, satisfying the equation (1) is nothing but the expansion containing infinite number of modes determined by these vertex operators. Both string fields and higher spin gauge fields in the equations (1) and (2) are known to be complicated objects to work with. Despite the fact that the higher spin theories in $A d S$ spaces can circumvent the restrictions imposed by the Coleman-Mandula's theorem, describing the gauge-invariant higher-spin interactions is a highly nontrivial problem since the gauge symmetry in these theories must be sufficiently powerful in order to eliminate unphysical degrees of freedom. The restrictions imposed by such a gauge symmetry make the construction of the interaction vertices in higher-spin theories a notoriously complicated problem. While there was some progress in classification of the higher-spin 3-vertices over recent years, the structure of the higher-order interactions (such as quartic interactions, presumably related to conformal blocks in dual CFT's) still remains obscure. The structure of these interactions is, however, crucial for our understanding of higher-spin extensions of the holography principle and non-supersymmetric formulation of $A d S / C F T$. 
At the same time, the string field theory still remains our best hope to advance towards background-independent formulation of string dynamics. This, in turn, holds the keys to understanding string theories in curved backgrounds, such as AdS. Such string theories are also crucially relevant to holography and gauge-string correspondence, however, little is known about them beyond the semiclassical limit .

Analytic solutions in string field theory appear to be one of the most crucial ingredients in order to approach such string theories in the SFT formalism, using the concept of background independence. To illustrate this, suppose a string field $\Psi_{0}$ is a solution of the equation (1). Then the form of (1) is invariant under the shift

$$
\Psi \rightarrow \tilde{\Psi}=\Psi+\Psi_{0}
$$

with the simultaneous shift of the BRST charge $Q \rightarrow \tilde{Q}$, so that $Q^{2}=\tilde{Q}^{2}=0$ and the new nilpotent charge $\tilde{Q}$ defined according to

$$
\tilde{Q} \Psi=Q \Psi+\Psi_{0} \star \Psi+\Psi \star \Psi_{0}
$$

for any $\Psi$. Then the new BRST charge $\tilde{Q}$ defines the new cohomology, different from that of the original charge $Q$, corresponding to string theory in a new background, depending on the structure of $\Psi_{0}$. The advantage of this approach is that, in principle, it allows to explore the string theory in new geometrical backgrounds (e.g. in a curved geometry, such as AdS) while technically using the operator products of the old string theory (say, in originally flat background) for the vertex operatos in the new BRST cohomology, defined by $\tilde{Q}$. This formalism is potentially more powerful than the first-quantized formalism, which is background-dependent and where the vertex operator description is essentially limited to the flat space-time and semiclassical limit of curved backgrounds. Unfortunately, however, the major obstacle is that identifying analytic solutions of the equation (1) is hard because of the complexity of the star product in (1). For this reason, there are not many known examples of analytic solutions having a clear physical interpretation. One of the most fascinating and well-known solutions, describing the nonperturbative tachyonic vacuum in string theory is of course the class of the Schnabl's solutions [7], later generalized in a number of important papers, in particular, such as [8], [9], [10], [11], [12] which were discovered several years ago and in particular used to prove the Sen's conjecture [13], [14] Since that remarkable paper by Schnable [7] there were many other interesting works describing the related SFT solutions, both in cubic theory and in Berkovits SFT theory 
[10], [11], [12], [15], [16], [17], [18] such as algebraic SFT solutions, the analytic solutions describing various nonperturbative processes such as D-brane translations. Despite that, classes of the SFT solutions, relevant to particular geometric backgrounds in string theory , in particular those that would allow us to advance towards consistent formulation of string theories in different space-time geometries, still mostly remain beyond our reach. One reason for this is that the star product in the equation (1) is hard to work with in practice [1], [2], [19], 220], 221], 22], [23], [15], [24], [25], [26], [27], [28]. In general, this product is quite different from the conventional Moyal product or the product in the Vasiliev's equations (2), although for certain restricted classes of string fields the star product of (1) can be mapped to the Moyal product [29], [30], [31]. In general, however, the star product involves the global conformal' transformations

$$
f_{k}^{N}(z)=e^{\frac{2 i \pi(k-1)}{N}}\left(\frac{1-i z}{1+i z}\right)^{\frac{2}{N}}
$$

that map the string fields living on separate worldsheets to $N$ wedges of a single disc. The behavior of generic string fields (containing all sorts of off-shell non-primary operators) under such global conformal transformations easily wobbles out of control beyond any low-level truncation, making it hard to evaluate the star product by straightforward computation of the correlators in OSFT. There are very few known exceptions to that, such as the wedge states or the special degenerate case of $\Psi$ constrained to primaries and their derivatives However, such fields form too small a subset in the space of all the operators. The known SFT solutions constrained to this subset do exist. However, with the exception of the Schnabl-related class of solutions, they are typically irrelevant to non-perturbative background deformations (see the discussion in the next section). At the same time, there exists a sufficiently large class of the operators (far larger than the class of the primary fields) which behaves in a rather compact and controllable way under (5), forming a closed subset of operators under the global conformal transformations. Typically, these operators have the form

$$
T^{(N)}=\sum_{k=1}^{N} \sum_{N \mid N_{1} \ldots N_{k}} \lambda_{N_{1} \ldots N_{k}}^{(N)} B^{\left(N_{1}\right)} \ldots B^{\left(N_{k}\right)}
$$

with the sum taken over the partitions of total conformal dimension $N$ of $T^{(N)}$ and with $B^{\left(N_{i}\right)}\left(\partial X, \partial^{2} X, \ldots, \partial^{N_{j}} X\right)$ being the Bell polynomials of rank $N_{j}$ in the worldsheet derivatives of string or superstring space-time coordinates or the ghost fields (we shall review the basic properties of these objects in the next section). The structure of the correlators of the 
operators of the form (6), analyzed in this work, as well as their transformation properties under (5) makes them natural candidates to test for the analytic solutions of (1). At the same time, it turns out that the structure constants of higher spin algebras in $A d S$ can be realized in terms of the OPE structure constants of the operators of the type (6). This makes a natural guess that the SFT solutions of the form (6) describe backgrounds with nonperturbative higher-spin configurations stemming from full interacting (to all orders) higher-spin theory in $A d S$. More precisely, this means the following. Suppose that somehow we manage to take a glimpse into full consistently interacting higher-spin theory and the higher spin interactions to all orders. Of course the Lagrangian of such a theory would be immensely complex, with all due restrictions imposed by the gauge invariance, with nonlocalities etc. One would also expect issues with unitarity as well, at least in backgrounds other than AdS. Assume, however, that we managed to identify such a higher-spin action and to solve the equations of motion, i.e. to find the higher-spin configuration minimizing this action. From the string theory point of view, such a background would correspond to a certain conformal fixed point, with vanishing $\beta$-functions of higher-spin vertex operators. An attempt to compute such $\beta$-functions straightforwardly would be hopeless, since that would require summing up contributions from all orders of the string perturbation theory. However, instead of computing the $\beta$-function, one can try to find an analytic solution describing the shift $Q \rightarrow \tilde{Q}_{H S}$ from the flat background to the one involving the nonperturbative higher spin configuration in AdS. To make a parallel to the Schnabl's solution for nonperturbative tachyonic background note that, from the on-shell string theory point of view, this solution describes the minimum of the tachyon potential stemming the tachyon's $\beta$-function, computed to all orders of the string perturbation theory. Given the SFT solution for nonperturbative higher-spin background, the cohomology of $\tilde{Q}_{H S}$ would then describe the physical properties of such a background. At the first glance, the structure of such a solution must be enormously complicated. Nevertheless, let's try to imagine its possible structure. The complete fully interacting higher-spin theory in $A d S_{d}$, no matter how complicated its Lanrangian might be, is largely determined by two objects: structure constants of the higher-spin algebras in $A d S$ and conformal blocks in the dual $C F T_{d-1}$. Moreover, as we shall argue in the next section, as far as the cubic SFT is concerned, for substantially large class of solutions the structure constants of the higher-spin algebra (more precisely, the enveloping of this algebra) alone constitute a sufficient information to control the solutions we are looking for. Thus, if one is able to find a class of SFT solutions determined by the structure constants of the HS algebra, this already would be a strong 
signal that it describes the higher spin background we are interested in. The rest of this paper is organized as follows. In the section 2 we shall discuss, as a warm-up example, a set of simple SFT solutions that involve the primary fields only and describe the perturbative background deformations. Remarkably, one particular example of these solutions is given by the discrete states in $c=1$ model where both the structure constants of the $A d S_{3}$ higher-spin algebra appear and the vertex operators are described in terms of products of the Bell polynomials of the type (4). In the section 3 we develop the OPE formalism for the Bell polynomials of string fields, evaluating their structure constants. We find that these structure constants can be obtained from simple generating function $G(x, y)$ of two variables, which series expansion is determined by coefficients related to $A d S_{3}$ structure constants. Next, we propose an ansatz of the form (6) solving (1). The solution is given by the certain composite function $F(G)$ satisfying certain defining relations derived in this paper and structurally can be thought of as an envelopping of the higher-spin algebra. In the concluding section we discuss the physical implications of our results and the generalizations relating analytic OSFT solutions to higher-spin algebras in higher dimensional AdS spaces.

\section{Structure Constants, Higher Spins and SFT solutions: a warm-up example}

One particularly simple and almost obvious example of a class of string fields solving (1) can be constructed as follows.

Let $V_{i}(z, p)(i=1, \ldots)$ be the set of all physical vertex operators in string theory in the cohomology of the original BRST charge $Q$ (primary fields of ghost number 1 and conformal dimension 0 ) and $\lambda^{i}(p)$ are the corresponding space-time fields (where $p$ is the momentum in space-time and we suppress the space-time indices for the brevity). Then the string field

$$
\Psi_{0}=\sum_{i} \lambda^{i} V_{i}
$$

is the solution of (1) provided that the zero $\beta$-function conditions:

$$
\beta_{\lambda^{i}}=0
$$

are imposed on the space-time fields in the leading order of the perturbation theory. This statement is easy to check. Indeed, the on-shell invariance conditions on $V_{i}$ imply $\left\{Q, \lambda^{i} V_{i}\right\}=\hat{L} \lambda^{i}=0$ where $\hat{L}$ is some differential operator (e.g. a Laplacian plus the square of mass) acting on $\lambda^{i}$. Next, since the operators are the dimension zero primaries, 
they are invariant under the transformations (5) and therefore the star product can be computed simply by using

$$
<<\Psi, \Psi \star \Psi>>=<\prod_{n=1}^{3} f_{n}^{3} \circ \Psi(0)>=\sum_{i, j, k} C_{i j k} \lambda^{i} \lambda^{j} \lambda^{k}
$$

where $C_{i j k}\left(p_{1}, p_{2}\right)$ are the structure constants in front of the simple pole in the OPE of the vertex operators:

$$
V_{i}\left(z_{1}, p_{1}\right) V_{j}\left(z_{2}, p_{2}\right) \sim\left(z_{1}-z_{2}\right)^{-1} C_{i j k}\left(p_{1}, p_{2}\right) V^{k}\left(\frac{1}{2}\left(z_{1}+z_{2}\right), p_{1}+p_{2}\right)
$$

Substituting (7), (9) into SFT equations of motion (1) then leads to the constraints on $\lambda^{i}$ space-time fields:

$$
\hat{L} \lambda^{i}+C_{j k}^{i} \lambda^{j} \lambda^{k}=0
$$

which are nothing but $\beta_{\lambda^{i}}=0$ equation (8) in the leading order. Note that the SFT solution (7) is entirely fixed by the leading order contribution to the $\beta$-function (which are completely determined by the 3-point correlation functions of the vertex operators) and does not depend on the higher-order corrections (related to the higher-point correlators). The higher order corrections to the $\beta$-function only appear upon the deformation (4) of the BRST charge related to the solution (7) which, in this case, simply reduces to $Q \rightarrow \tilde{Q}=Q+\sum \lambda_{i} V^{i}$. The 4-point functions of the $V_{i}$ vertex operators will then determine the solution of the equation (1) with $Q$ replaced by $\tilde{Q}$. This, in turn, will lead to the further shift of $\tilde{Q}$ in the next order etc., so the whole procedure can be performed order by order. The physical meaning of these deformations is also quite clear: they define, order by order, the perturbative changes of the background caused by the $\mathrm{RG}$ flows from the original conformal point (corresponding to flat background) to the new fixed point (corresponding to a certain solution of the low-energy effective equations of motion). Physically, far more interesting is of course the case when the operators entering (7) are no longer the primaries of any fixed conformal dimensions and are off-shell, but still solve (1) with the constraints of the type (11). Then, $\lambda$ describes the background which is beyond the reach of the conventional string perturbation theory while the $C$-constants describe the new $2 d$ CFT related to this non - perturbative background change. This is precisely the type of the higher-spin related SFT solution we will be looking for. The instructive point here (which 
follows from the above discussion) is that, if we start with the SFT equation (1) with the unperturbed BRST charge of the bosonic theory:

$$
Q=\oint d z\{c T-b c \partial c\}=\oint d z\left\{-\frac{1}{2} \partial X_{m} \partial X^{m}+b c \partial c\right\}
$$

the higher-spin solution we are searching for shouldn't depend on higher-point correlators or the conformal blocks, but only on the structure constants of the higher-spin algebra. The final remark we shall make before moving further regards the appearance of the higher spin algebra in the SFT solution of the type (7) at the perturbative level, as well as the appearance of the Bell polynomials as the operators realizing this algebra. Consider the noncritical open one-dimensional bosonic string theory (also known as the $c=1$ model). It is well-known that this string theory does not contain a photon in the massless spectrum, however, due to the $S U(2)$ symmetry at the self-dual point, it does contain the $S U(2)$ multiplet of the discrete states which are physical at integer or half-integer momentum values only and become massless upon the Liouville dressing. To obtain the vertex operators for these states, consider the $S U(2)$ algebra generated by

$$
\begin{array}{r}
T_{ \pm}=\oint d z e^{ \pm i X \sqrt{2}} \\
T_{0}=\frac{i}{\sqrt{2}} \partial X
\end{array}
$$

where $X$ is a single target space coordinate and the dressed BRST-invariant highest weight vector

$$
V_{l}=\int d z e^{(i l X+(l-1) \varphi) \sqrt{2}}
$$

where $\varphi$ is the Liouville field and $l$ is integer or half-integer. The $\mathrm{SU}(2)$ multiplet of the operators is then obtained by repeatedly acting on $V_{l}$ with the lowering operator $T_{-}$of $\mathrm{SU}(2)$ :

$$
\begin{array}{r}
U_{l \mid m}=T_{-}^{l-m} V_{l} \\
-l \leq m \leq l
\end{array}
$$

The dressed $U_{l \mid m}$ operators are the physical operators (massless states) of the $c=1$ model and are the worldsheet integrals of primary fields of dimension one (equivalently, the primaries of dimension 0 at the unintegrated $b-c$ ghost number 1 picture)

Manifest expressions for the $U_{l \mid m}$ vertex operators are complicated, however, their structure constants have been deduced by [32], [33] by using symmetry arguments. One has

$$
U_{l_{1} \mid m_{1}}(z) U_{l_{2} \mid m_{2}}(w) \sim(z-w)^{-1} C\left(l_{1}, l_{2}, l_{3} \mid m_{1}, m_{2}, m_{3}\right) f\left(l_{1}, l_{2}\right) U_{l_{3}, m_{3}}
$$


where the $S U(2)$ Clebsch-Gordan coefficients are fixed by the symmetry while the function of Casimir eigenvalues $f\left(l_{1}, l_{2}\right)$ is nontrivial and was deduced to be given by [32], [33]

$$
f\left(l_{1}, l_{2}\right)=\frac{\sqrt{l_{1}+l_{2}}\left(2 l_{1}+2 l_{2}-2\right) !}{\sqrt{2 l_{1} l_{2}}\left(2 l_{1}-1\right) !\left(2 l_{2}-1\right) !}
$$

Remarkably, these structure constants coincide (up to a simple field redefinition) exactly with those of $w_{\infty}$ wedge, defining the asymptotic symmetries of the higher spin algebra in $A d S_{3}$ in a certain basis, computed in a rather different context [34]. Thus the primaries (15) are connected to a vertex operator realization of $A d S_{3}$ higher-spin algebra. The related OSFT solution is then constructed similarly to the previous one. It is given simply by

$$
\Psi=\sum_{l, m} \lambda^{l \mid m} U_{l \mid m}
$$

with the constants $\lambda^{l \mid m}$ satisfying the $\beta$-function condition

$$
S_{l_{1} m_{1} \mid l_{2} m_{2}}^{l_{3} \mid m_{3}} \lambda^{l_{1} m_{1}} \lambda^{l_{2} m_{2}}=0
$$

where $S_{l_{1} m_{1} \mid l_{2} m_{2}}^{l_{3} \mid m_{3}}=C\left(l_{1}, l_{2}, l_{3} \mid m_{1}, m_{2}, m_{3}\right) f\left(l_{1}, l_{2}\right)$ are the $A d S_{3}$ higher spin algebra's structure constants. As previously, this solution describes the perturbative background's change

$$
Q \rightarrow \tilde{Q}=Q+\sum_{l, m} \lambda^{l \mid m} U_{l \mid m}
$$

- The higher order contributions to the $\beta$-function then will appear in the SFT solutions with $Q$ replaced with $\tilde{Q}$ etc. Our particular goal is, roughly speaking, to find the off-shell analogues of the string field (18) solving the SFT equation of motion (1), with $\lambda$-constants satisfying the constraints related to the structures of the higher-spin algebras.

For that, it is first instructive to investigate the manifest form of the operators (note that in [32], 33]) the structure constants were computed from the symmetry arguments, without pointing out the explicit form of the operators). Taking the highest weight vector $V_{l}$ (14) and applying $T_{-}$using the OPE

$$
e^{-i X \sqrt{2}}(z) e^{(i l X+(l-1) \varphi) \sqrt{2}}(w)=\sum_{k=0}^{\infty}(z-w)^{k-2 l} B_{-i \sqrt{2} X}^{(k)}: e^{(i(l-1) X+(l-1) \varphi) \sqrt{2}}:(w)
$$

we obtain

$$
U_{l \mid l-1}=T_{-} V_{l}=\oint d w: B_{-i \sqrt{2} X}^{(2 l-1)} e^{(i(l-1) X+(l-1) \varphi) \sqrt{2}}:(w)
$$


Here $B_{f(z)}^{(n)} \equiv B^{(n)}\left(\partial_{z} f, \ldots, \partial_{z}^{n} f\right)$ are the rank $n$ normalized Bell polynomials in the derivatives of $f$, defined according to

$$
\begin{array}{r}
B^{(n)}\left(\partial_{z} f, \ldots, \partial_{z}^{n} f\right)=\left.B^{(n)}\left(x_{1}, \ldots x_{n}\right)\right|_{x_{k} \equiv \partial^{k} f ; 1 \leq k \leq n} \\
=\sum_{k=1}^{n} B_{n \mid k}\left(x_{1}, \ldots x_{n-k+1}\right)
\end{array}
$$

where $B_{n \mid k}\left(x_{1}, \ldots x_{n-k+1}\right)$ are the normalized partial Bell polynomials defined according to

$$
B_{n \mid k}\left(x_{1}, \ldots x_{n-k+1}\right)=\sum_{p_{1}, \ldots p_{n-k+1}} \frac{1}{p_{1} ! \ldots p_{n-k+1} !} x_{1}^{p_{1}}\left(\frac{x_{2}}{2 !}\right)^{p_{2}} \ldots\left(\frac{x_{n-k+1}}{(n-k+1) !}\right)^{p_{n-k+1}}
$$

with the sum taking over all the combinations of non-negative $p_{j}$ satisfying

$$
\begin{gathered}
\sum_{j=1}^{n-k+1} p_{j}=k \\
\sum_{j=1}^{n-k+1} j p_{j}=n
\end{gathered}
$$

(note that the standard Bell polynomials $P^{(n)}$ are related to the normalized ones as $P^{(n)}=$ $n ! B^{(n)}$; similarly for the partial Bell polynomials) To calculate the next vertex operator, $U_{l \mid l-2}=T_{-} U_{l \mid l-1}$ one needs to point out, apart from the OPE (21), the OPE between Bell polynomials of the $X$-derivatives and the exponents of $X$ as well. Using the definitions (23)-(25), it is straightforward to deduce the identity

$$
B_{\alpha X}^{(n)}(z) e^{\beta X}(w)=\sum_{k=0}^{n}(z-w)^{-k} \frac{\Gamma(-\alpha \beta+1)}{k ! \Gamma(-\alpha \beta+1-k)}: B_{\alpha X}^{(n-k)}(z) e^{\beta X}(w):
$$

where $\alpha$ and $\beta$ are some numbers and $\Gamma$ is the Euler's gamma-function. Note that this is the double point OPE (sufficient for our purposes), i.e. accounting only for the contractions between $B_{\alpha X}^{(n)}$ and $e^{\beta X}(w)$, but not for the expansions of any of them around some fixed point (such as $z, w$ or a midpoint).

Using (21), (26), it is then straightforward to obtain:

$$
U_{l \mid l-2}=2 ! \oint d w: e^{(i(l-2) X+(l-1) \varphi) \sqrt{2}}\left(B_{-i \sqrt{2} X}^{(2 l-1)} B_{-i \sqrt{2} X}^{(2 l-3)}-\left(B_{-i \sqrt{2} X}^{(2 l-2)}\right)^{2}\right):(w)
$$

This operator is given by the exponent multiplied by the quadratic combination of the Bell polynomials with ranks $B^{\left(2 l-k_{j}\right)} ; j=1,2$ with $k_{1}+k_{2}$ being the length 2 partition 
of $2^{2}=4$ with $1 \leq k_{1,2} \leq 2 \times 2-1=3$. It is straightforward to continue this sequence of transformations by $T_{-}$to identify the manifest expressions for all the vertex operators. For arbitrary $U_{l \mid l-m}(1 \leq m \leq l)$ we obtain

$$
U_{l \mid l-m}=m ! \oint d w e^{(i(l-m) X+(l-1) \varphi) \sqrt{2}} \sum_{m^{2} \mid k_{1} \ldots k_{m}}(-1)^{\pi\left(k_{1}, \ldots, k_{m}\right)} B_{-i \sqrt{2} X}^{\left(2 l-k_{1}\right)} B_{-i \sqrt{2} X}^{\left(2 l-k_{2}\right)} \ldots B_{-i \sqrt{2} X}^{\left(2 l-k_{m}\right)}
$$

with the sum taken over all the ordered length $m$ partitions of $m^{2}=k_{1}+\ldots+k_{m}$ with $1 \leq k_{1} \leq \ldots \leq k_{m} \leq 2 m-1$ and with the parity $\pi\left(k_{1}, \ldots, k_{m}\right)$ of each partition defined as follows. Consider a particular partition of $m^{2}: k_{1} \leq k_{2} \ldots \leq k_{m}$. By permutation we shall call any exchange between two neighbouring elements of the partition with one unit that does not break the order of the partition, e.g.

$$
\begin{array}{r}
\left\{k_{1} \leq \ldots k_{i-1} \leq k_{i} \leq k_{i+1} \leq k_{i+2} \leq \ldots \leq k_{m}\right\} \\
\rightarrow\left\{k_{1} \leq \ldots k_{i-1} \leq\left(k_{i} \pm 1\right) \leq\left(k_{i+1} \mp 1\right) \leq k_{i+2} \leq \ldots \leq k_{m}\right\}
\end{array}
$$

Then $\pi\left(k_{1}, \ldots, k_{m}\right)$ for any length $m$ partition of $m^{2}$ is the minimum number of permutations needed to obtain the partition $m^{2}=k_{1}+\ldots .+k_{m}$ from the reference partition $m^{2}=$ $1+3+5+\ldots+(2 m-1)$. Note that, possibly up to an overall sign change of $U_{l \mid m}$, any partition can be chosen as a reference partition. One particular lesson that we learn from (28) is that combinations of the objects of the type $\sum_{\left\{n_{1} \ldots n_{k}\right\} ; N=n_{1}+\ldots+n_{k}} \alpha_{n_{1} \ldots n_{k}} \prod_{j=1}^{k} B^{\left(n_{j}\right)}$ form a basis for the operator realization of the higher-spin algebra. As we will see below, this is not incidental, as the products of the Bell polynomials naturally realize $w_{\infty}$ and envelopings of $S U(2)$. In general, they are not primary fields, except for some very special choices of the $\alpha_{n_{1} \ldots n_{k}}$ coefficients in the summation over the partitions (18). (strictly speaking, the products of Bell polynomials in (18), (19) are the primaries only for $m=l$; otherwise they must be dressed with the exponents). Two important numbers characterizing these objects are $N$ and $k$ (total conformal dimension and the partition length).

The ansatz for the solution in $D$-dimensional string field theory that we propose is the following. Define the generating function for the Bell polynomials:

$$
H(B)=\sum_{n=1}^{\infty} h_{n} B_{\vec{\alpha} \vec{X}}^{(n)} \equiv \sum_{n=1}^{\infty} \frac{h_{n} P_{\vec{\alpha} \vec{X}}^{(n)}}{n !}
$$

where $P^{(n)}$ are the standard (non-normalized) Bell polynomials in the derivatives of $\vec{\alpha} \vec{X}$, $h_{n}$ are some coefficients, defining the associate characteristic function

$$
H(x)=\sum_{n} \frac{h_{n} x^{n}}{n !}
$$


This function is convenient to use in order to perform various operations with $H(B)$; e.g. the derivative function $H^{\prime}(B)$ can be obtained by differentiating $H(x)$ over $x$ and then replacing $\frac{x^{n}}{n !} \rightarrow B_{\vec{\alpha} \vec{X}}^{(n)}$ in the expansion series obtained by differentiation. Next, define another characteristic function

$$
G(x)=\sum_{n=0}^{\infty} \frac{g_{n} x^{n}}{n !}
$$

Then the composite function $G(H(B))$ generates the products of Bell polynomial operators according to the Faa de Bruno formula (which is easy to check by simple straightforward computation):

$$
\begin{array}{r}
G(H(B))=\sum_{n=0}^{\text {infty }} \frac{g^{n}}{n !} \sum_{N=n}^{\infty} N ! \sum_{N \mid k_{1} \ldots k_{n}} h_{k_{1}} \ldots . h_{k_{n}} B_{\vec{\alpha} \vec{X}}^{\left(k_{1}\right)} \ldots B_{\vec{\alpha} \vec{X}}^{\left(k_{n}\right)} \sigma^{-1}\left(k_{1}, \ldots k_{n}\right) \\
=\sum_{n=0}^{\infty} \frac{g^{n}}{n !} \sum_{N=n}^{\infty} B_{N \mid n}\left(h_{1} B_{\vec{\alpha} \vec{X}}^{(1)}, \ldots, h_{N-n+1} B^{\left.(N-n+1)_{\vec{\alpha} \vec{X}}\right)}\right.
\end{array}
$$

where $\sum_{N \mid k_{1} \ldots k_{n}}$ stands for the summation over ordered length $n$ partitions of $N(0<$ $\left.k_{1} \leq k_{2} \ldots \leq k_{n}\right)$ and the sigma-factor

$$
\sigma\left(k_{1}, \ldots, k_{n}\right)=q_{k_{1}} ! \ldots q_{k_{n}} !
$$

is the product of the multiplicities of the elements $k_{j}$ of the partition. ( note that each $q_{k_{j}}$ elements $k_{j}$ entering the partition give rise to the single factor of $q_{k_{j}}$ ! in the $\sigma^{-1}$ denominator in (33)) We will be looking for the ansatz SFT solution in the form (35), that is,

$$
\Psi=G(H(B))
$$

and our goal is to determine the coefficients $h_{n}$ and $g_{n}$ (more precisely, the defining constraints on these coefficients imposed by the SFT equations of motion). $\vec{\alpha}$ is some parameter which a priori is not fixed; however, we shall see below that the star product for $\Psi$ is drastically simplified if the tachyon-like constraint: $\alpha^{2}=-2$ is imposed on $\vec{\alpha}$ and it is precisely this simplification that ultimately makes it possible to formulate the SFT solutions in terms of the functional relations for $G(H)$. As it is clear from (33)-(35), the SFT ansatz that we propose is given by the series in the partial Bell polynomials of the Bell polynomials in the target space fields. An essential property of these objects is that their operator algebra realizes the enveloping of $S U(2)$ with the enveloping parameter related to $\alpha^{2}$. In particular, a simple pole in the OPE of these objects leads to classical $w_{\infty}$ algebra 
of area-preserving diffeomorphisms, while the complete OPE generates the full enveloping ( the explicit OPE structure will be given below). This is where the connection with the higher spin algebra enters the game. To prepare for the analysis of (1) using the ansatz (33)-(35), in the next section we shall analyze the conformal transformation properties, operator products and the correlators of the vertex operators involving the Bell polynomials and their products.

\section{CFT Properties of SFT ansatz}

The first important building block in our construction of the SFT solution is the analysis of the conformal field theory properties of operators constructed out of products of the Bell polynomials in the target space fields. The first step is to determine the transformation laws for the operators in the sum (33). We start from the infinitezimal transformation of a single Bell polynomial. First, we need to evaluate the operator product of the stress tensor with $B_{\vec{\alpha} \vec{X}}^{(n)}$. This can be done by using the identity

$$
\partial_{z}^{n} e^{\vec{\alpha} \vec{X}}(z)=n ! B_{\vec{\alpha} \vec{X}}^{(n)} n e^{\vec{\alpha} \vec{X}}
$$

Then one can deduce the infinitezimal conformal transformation of $B_{\vec{\alpha} \vec{X}}^{(n)}$ with the generator

$$
\oint d z \epsilon(z) T(z)=-\frac{1}{2} \oint d z \epsilon(z) \partial X_{m} \partial X^{m}(z)
$$

by using the identity

$$
\delta_{\epsilon}\left(\partial_{z}^{n} e^{\vec{\alpha} \vec{X}}\right)=n !\left(\delta_{\epsilon} B_{\vec{\alpha} \vec{X}}^{(n)}\right) e^{\vec{\alpha} \vec{X}}+n ! B_{\vec{\alpha} \vec{X}}^{(n)}\left(\delta_{\epsilon} e^{\vec{\alpha} \vec{X}}\right)+\delta_{\epsilon}(\text { overlap })
$$

with $\delta_{\epsilon}$ (overlap) accounting for the contribution in which one of the $\partial X$ 's of $T$ is contracted with $B^{(n)}$ and another with the exponent. Using the manifest expression for $B_{\psi}^{(n)}$ :

$$
B_{\psi}^{(n)}=\sum_{l} \sum_{n \mid p_{1} \ldots p_{l}} \frac{\left(\partial^{p_{1}} \psi\right)^{m_{1}} \ldots\left(\partial^{p_{l}} \psi\right)^{m_{l}}}{p_{1} ! \ldots p_{k} ! m_{1} ! \ldots m_{k} !}
$$

where $\psi=\vec{\alpha} \vec{X}$ and the sum is taken over the ordered partitions

$$
\begin{array}{r}
n=\sum_{j=1}^{l} m_{j} p_{j} \\
k=\sum_{j=1}^{l} m_{j} \\
1 \leq k \leq n ; 1 \leq l \leq k \\
p_{1}<p_{2}<\ldots<p_{l}
\end{array}
$$


it is straightdorward to establish the OPE:

$$
\partial X_{m}(z) B_{\psi}^{(n)}(w)=-\alpha_{m} \sum_{k=1}^{n}(z-w)^{-k-1} B_{\psi}^{(n-k)}(w)+\text { regular }
$$

Using the OPE (41) it is straightforward to compute the overlap transformation and to deduce the OPE between the stress-energy tensor and $B_{\psi}^{(n)}$ with the result given by

$$
\begin{gathered}
T(z) B_{\psi}^{(n)}(w)=(z-w)^{-1} \partial B_{\psi}^{(n)}(w)+n(z-w)^{-2} B_{\psi}^{(n)}(w)+ \\
+\sum_{k=2}^{n+1}(z-w)^{-k-1}\left(n+1+\alpha^{2}-\frac{1}{2}\left(\alpha^{2}+2\right) k\right) B_{\psi}^{(n-k+1)}(w)
\end{gathered}
$$

Note that the coefficients in front of $B_{\psi}^{(n-k+1)}(w)$ do not depend on $k$ when $\vec{\alpha}$ satisfies the tachyon-like condition $\alpha^{2}=-2$. This drastically simplifies the problem to determine the behaviour of $B^{(n)}$ under the global conformal transformations in the SFT equations, which infinitezimal form is defined by the OPE (42). Using the OPE's (41), (42), it is now straightforward to deduce the OPE of $T(z)$ with the product of any number $q$ of the Bell polynomials of the target space fields, which will be the main building block for the SFT solutions that we are looking for. It is convenient to introduce the notation:

$$
\begin{array}{r}
R_{N}^{n_{1} \ldots n_{q}}=\prod_{j=1}^{q} B_{\psi}^{\left(n_{j}\right)}(w) \\
N=\sum_{j} n_{j}
\end{array}
$$

We have:

$$
\begin{array}{r}
T(z) R_{N}^{n_{1} \ldots n_{q}}(w)=\sum_{j=1}^{q} \sum_{k_{j}=2}^{n_{j}+1}(z-w)^{-k_{j}-1}\left(n_{j}+1+\alpha^{2}-\frac{1}{2}\left(\alpha^{2}+2\right) k_{j}\right) R_{N-n_{j}}^{\left.n_{1} \ldots n_{q}\right|_{j}} B_{\psi}^{\left(n_{j}-k_{j}+1\right)} \\
-\alpha^{2} \sum_{l, m=1 ; l<m}^{q}(z-w)^{-k_{l}-k_{m}}\left(R_{N-n_{l}-n_{m}}^{\left.n_{1} \ldots n_{q}\right|_{l, m}}\right) B_{\psi}^{\left(n_{l}-k_{l}+1\right)} B_{\psi}^{\left(n_{m}-k_{m}+1\right)} \\
+N(z-w)^{-2} R_{N}^{n_{1} \ldots n_{q}}(w)+(z-w)^{-1} \partial R_{N}^{n_{1} \ldots n_{q}}(w)
\end{array}
$$

where $\left.n_{1} \ldots n_{q}\right|_{j}$ stands for the set of $q-1$ indices with $n_{j}$ excluded, similarly for $\left.n_{1} \ldots n_{q}\right|_{l, m}$. Given the OPE (44) it is straightforward to obtain the infinitezimal the transformation law for $c R_{N}^{n_{1} \ldots n_{q}}$ : 
We have:

$$
\begin{array}{r}
\delta_{\epsilon}\left(c R_{N}^{n_{1} \ldots n_{q}}\right)(w)=\sum_{j=1}^{q} \sum_{k_{j}=2}^{n_{j}+1} \frac{\partial^{k_{j}} \epsilon}{k_{j} !}\left(n_{j}+1+\alpha^{2}-\frac{1}{2}\left(\alpha^{2}+2\right) k_{j}\right) c R_{N-n_{j}}^{\left.n_{1} \ldots n_{q}\right|_{j}} B_{\psi}^{\left(n_{j}-k_{j}+1\right)} \\
-\alpha^{2} \sum_{l, m=1 ; l<m}^{q} \frac{\partial^{k_{l}+k_{m}-1} \epsilon}{\left(k_{l}+k_{m}-1\right) !} c R_{N-n_{l}-n_{m}}^{\left.n_{1} \ldots n_{q}\right|_{l, m}} B_{\psi}^{\left(n_{l}-k_{l}+1\right)} B_{\psi}^{\left(n_{m}-k_{m}+1\right)} \\
+(N-1) \partial \epsilon R_{N}^{n_{1} \ldots n_{q}}(w)+\epsilon \partial\left(c R_{N}^{n_{1} \ldots n_{q}}\right)(w)
\end{array}
$$

Now we have to establish transformation law for $c R_{N}^{n_{1} \ldots n_{q}}$ under $z \rightarrow f(z)$, necessary to compute the correlators in the string field theory equations of motion. This can be deduced from two conditions: first, the global transformation should reproduce (45) for $f(z)=z+\epsilon$. Second, the form of the global transformation must be preserved under the composition. As it is well-known, in case of simplest non-primary field, such as the stressenergy tensor, this leads to the appearance of the Schwarzian derivative of $f(z)$ which is in fact the degree 2 Bell polynomial in $\log \left(f^{\prime}(z)\right)$ :

$$
S(f(z))=2 B_{2}\left(-\frac{1}{2} \log \left(f^{\prime}\right)\right)
$$

This is not a coincidence since for a large class of non-primaries in $C F T$ the higher degree Bell polynomials correspond to the higher derivative extensions of the Schwarzian derivative in conformal transformations. Note that the Bell polynomials of the logarithms of functions defining global conformal transformation satisfy the following composition identity:

$$
B^{(n)}\left(\log \left(\frac{d}{d x} f(g(x))\right)\right)=\sum_{k} B^{(n-k)}\left(\log \left(f^{\prime}(g)\right)\right) B^{(k)}\left(\log \left(g^{\prime}(x)\right)\right)
$$

making them natural objects present in global conformal transformations. The global conformal transformations of the Bell polynomials, consistent with the infinitezimal transformations (45) are deduced to be given by

$$
\begin{array}{r}
c R_{N}^{n_{1} \ldots n_{q}}(z) \rightarrow_{z \rightarrow f(z)}\left(\frac{d f}{d z}\right)^{N-1} c R_{N}^{n_{1} \ldots n_{q}}(f(z)) \\
-\sum_{j=1} \sum_{k_{j}=2}^{n_{j}+1} \frac{1}{k_{j}}\left(\frac{d f}{d z}\right)^{N-k_{j}} B^{\left(k_{j}-1\right)}\left(-\left(n_{j}+1+\alpha^{2}-\frac{1}{2}\left(\alpha^{2}+2\right) k_{j}\right) \log \left(\frac{d f}{d z}\right)\right) \\
\times c R_{N-n_{j}}^{\left.n_{1} \ldots n_{q}\right|_{j}} B_{\psi}^{\left(n_{j}-k_{j}+1\right)} \\
+\sum_{l, m=1 ; l<m}^{q} \frac{1}{\left(k_{l}+k_{m}-1\right)}\left(\frac{d f}{d z}\right)^{N-k_{l}-k_{m}+1} B_{k_{l}+k_{m}-2}\left(-\alpha^{2} \log \left(\frac{d f}{d z}\right)\right) \\
\times c R_{N-n_{l}-n_{m}}^{\left.n_{1} \ldots n_{q}\right|_{l, m}} B_{\psi}^{\left(n_{l}-k_{l}+1\right)} B_{\psi}^{\left(n_{m}-k_{m}+1\right)}
\end{array}
$$


For our purposes, we shall need to compute the values of the Bell polynomials in the transformation law (48) for the functions $I(z)=-\frac{1}{z}$ (in the kinetic term of the SFT action) and $g \circ f_{k}^{3}(z)$ at $z=0$ where $f_{k}^{3}$ defined in (5) map the string worldsheets of the cubic theory to the wedges of the disc and

$$
g(z)=i \frac{1-z}{1+z}
$$

further maps this disc to the half-plane, so that

$$
\begin{array}{r}
g \circ f_{1}^{3}(0)=0 \\
g \circ f_{2}^{3}(0)=\sqrt{3} \\
g \circ f_{3}^{3}(0)=-\sqrt{3}
\end{array}
$$

For that, we shall use the fact that if $\left\{a_{n}\right\}$ are the coefficients in the series expansion of any function $f(x)=\sum_{n=1} \frac{a_{n} x^{n}}{n !}($ assume $f(0)=0)$, then $e^{f(x)}=1+\sum_{n=1}^{\infty} B^{(n)}\left(a_{1}, \ldots, a_{n}\right) x^{n}$. From now on, to abbreviate things, we shall restrict ourselves to the case $\alpha^{2}=-2$, relevant to our SFT solution. We start from $B^{(k)}\left(\kappa \log \left(I^{\prime}(z)\right)\right)$ where, in particular, $\kappa=1-n_{j}$ in the first group of terms in (48) and $\kappa=-\alpha^{2}=2$ in the second. Then

$$
B^{(n)}\left(\kappa \log \left(I^{\prime}(z)\right)\right)=z^{-n} B^{(n)}\left(2 \kappa,-2 \kappa, \ldots(-1)^{n} 2(n-1) ! \kappa\right)
$$

The Bell polynomial on the right-hand side is then identified with the $n$ 'th expansion coefficient of the exponent of $-2 \kappa \log z$, i.e. of $z^{-2 \kappa}$ Therefore

$$
B^{(n)}\left(\kappa \log \left(I^{\prime}(z)\right)\right)=\frac{\Gamma(1-2 \kappa) z^{-n}}{n ! \Gamma(1-2 \kappa-n)}
$$

Next, we need the values of the Bell polynomials $B^{(n)}\left(\log \frac{d f(z)}{d z}\right)$ with $f(z)=g \circ f_{k}^{3}(z)$ at $z=0$. Straightforward calculation gives the result

$$
\left.B^{(n)}\left(\kappa \log \frac{d f(z)}{d z}\right)\right|_{z=0}=B^{(n)}\left(\beta_{1} \ldots \beta_{k} \ldots \beta_{n}\right)
$$

with

$$
\beta_{k}=\kappa\left(\frac{5}{3}(-i)^{n}+\frac{1}{3}(i)^{n}-\left(\frac{1}{2}\right)^{n-1}\right)(n-1) !
$$

for $g \circ f_{1}^{3}(z)$, 


$$
\beta_{k}=\kappa\left(\frac{5}{3}(-i)^{n}+\frac{1}{3}(i)^{n}+2 e^{\frac{2 i \pi n}{3}}\right)(n-1) !
$$

for $g \circ f_{2}^{3}(z)$ and

$$
\beta_{k}=\kappa\left(\frac{5}{3}(-i)^{n}+\frac{1}{3}(i)^{n}+2 e^{\frac{-2 i \pi n}{3}}\right)(n-1) !
$$

for $g \circ f_{3}^{3}(z)$. Accordingly, these Bell polynomials are identified with the expansion series of

$$
\begin{array}{r}
h_{1}(z)=(1+i z)^{-\frac{5}{3} \kappa}(1-i z)^{-\frac{1}{3} \kappa}\left(1+\frac{z}{2}\right)^{-2 \kappa} \\
h_{2}(z)=(1+i z)^{-\frac{5}{3} \kappa}(1-i z)^{-\frac{1}{3} \kappa}\left(1-e^{\frac{2 i \pi}{3}} z\right)^{-2 \kappa} \\
h_{3}(z)=(1+i z)^{-\frac{5}{3} \kappa}(1-i z)^{-\frac{1}{3} \kappa}\left(1-e^{\frac{-2 i \pi}{3}} z\right)^{-2 \kappa}
\end{array}
$$

for $g \circ f_{1}^{3}, g \circ f_{2}^{3}$ and $g \circ f_{3}^{3}$ respectively. Accordingly, the values of the Bell polynomials are given by

$$
\begin{aligned}
& \left.B^{(n)}\left(\kappa \log \left(\frac{d}{d z} g \circ f_{1}^{3}(z)\right)\right)\right|_{z=0} \\
& =\sum_{k, l, m \mid k+l+m=n} \frac{e^{\frac{i \pi}{2}(k-l)} 2^{-m}}{k ! l ! m !} \frac{\Gamma\left(1-\frac{5}{3} \kappa\right) \Gamma\left(1-\frac{1}{3} \kappa\right) \Gamma(1-2 \kappa)}{\Gamma\left(1-\frac{5}{3} \kappa-k\right) \Gamma\left(1-\frac{1}{3} \kappa-l\right) \Gamma(1-2 \kappa-m)} \\
& \left.B^{(n)}\left(\kappa \log \left(\frac{d}{d z} g \circ f_{2}^{3}(z)\right)\right)\right|_{z=0} \\
& =\sum_{k, l, m \mid k+l+m=n} \frac{e^{i \pi\left(\frac{1}{2}(k-l)+\frac{2 m}{3}\right)}}{k ! l ! m !} \frac{\Gamma\left(1-\frac{5}{3} \kappa\right) \Gamma\left(1-\frac{1}{3} \kappa\right) \Gamma(1-2 \kappa)}{\Gamma\left(1-\frac{5}{3} \kappa-k\right) \Gamma\left(1-\frac{1}{3} \kappa-l\right) \Gamma(1-2 \kappa-m)} \\
& \left.B^{(n)}\left(\kappa \log \left(\frac{d}{d z} g \circ f_{3}^{3}(z)\right)\right)\right|_{z=0} \\
& =\sum_{k, l, m \mid k+l+m=n} \frac{e^{i \pi\left(\frac{1}{2}(k-l)-\frac{2 m}{3}\right)}}{k ! l ! m !} \frac{\Gamma\left(1-\frac{5}{3} \kappa\right) \Gamma\left(1-\frac{1}{3} \kappa\right) \Gamma(1-2 \kappa)}{\Gamma\left(1-\frac{5}{3} \kappa-k\right) \Gamma\left(1-\frac{1}{3} \kappa-l\right) \Gamma(1-2 \kappa-m)}
\end{aligned}
$$

with the sums taken over the unordered partitions of $n=k+l+m$. These relations altogether fully determine the transformation properties of our string field ansatz, including the star product.

The final step to make before actually computing the SFT correlators is to point out the operator product rules involving the Bell polynomial operators and their blocks. We will do this in the next section, in particular deriving an analogue of the generalized Wick's theorem for the Bell polynomial operators and pointing the relevance of their correlators to the structure constants of the higher-spin algebra. 


\section{Bell Polynomial Operators: Operator Products and Correlators}

The most crucial building block in our computations involves the OPE rules for the operators of the SFT ansatz (33)-(35) which we will establish in this section. Ultimately, it turns out that it is precisely the structure of these OPE rules which makes it possible to work out the SFT solution and, moreover, to relate it to the higher spin algebra.

We start from the simplest OPE between $B_{\vec{\alpha} \vec{X}}^{(N)}(z)$ and $B_{\vec{\beta} \vec{X}}^{(m)}(w)$. This doesn't turn out to be an easy OPE to compute. The manifest expressions (23)-(24) for the Bell polynomials do not appear to be very helpful. Nevertheless, there are some observations to simplify the computation. First of all, the OPE has to preserve the conformal transformation structure (48) of the Bell polynomial operators. This suggests that the OPE must have the structure

$$
B_{\vec{\alpha} \vec{X}}^{(N)}(z) B_{\vec{\beta} \vec{X}}^{(M)}(w)=\left.\sum_{n=0}^{N} \sum_{m=0}^{M}(z-w)^{-n-m} \lambda^{N \mid M}\right|_{m \mid n}: B_{\vec{\alpha} \vec{X}}^{(N-n)}(z) B_{\vec{\beta} \vec{X}}^{(M-m)}(w):
$$

(again, for the brevity we consider the double point OPE here, just as was explained above). In other words, the Bell polynomial structure (48) of the operators is preserved by (59). The next helpful hint comes from the identity (36) relating the Bell operators to the derivatives of the exponents and from analyzing the correlator

$$
\begin{array}{r}
<B_{\vec{\alpha} \vec{X}}^{(N)} e^{\vec{\alpha} \vec{X}}(z) B_{\vec{\beta} \vec{X}}^{(M)} e^{\vec{\beta} \vec{X}}(w) \\
=\frac{1}{N ! M !} \partial_{z}^{N} \partial_{w}^{M}<e^{\vec{\alpha} \vec{X}}(z) e^{\vec{\beta} \vec{X}}(w)>=(z-w)^{-\vec{\alpha} \vec{\beta}-N-M} \frac{\Gamma(1-\vec{\alpha} \vec{\beta})}{N ! M ! \Gamma(1-\vec{\alpha} \vec{\beta}-M-N)}
\end{array}
$$

This correlator can be computed in two equivalent ways: one either starts with applying the OPE (26) of the Bell polynomials with the exponents and then contracting the remaining derivatives of $X$ between themselves in each of the OPE terms - or, alternatively, starting with the OPE (59) between the Bell polynomials, containing the unknown $\lambda$-constants and then contracting the remaining derivatives of $\mathrm{X}$ in each of the operators with the opposite exponent. Comparison of these two expressions identifies the remarkably simple OPE structure:

$$
B_{\vec{\alpha} \vec{X}}^{(N)}(z) B_{\vec{\beta} \vec{X}}^{(N)}(w)=<B_{\vec{\alpha} \vec{X}}^{(n)}(z) B_{\vec{\beta} \vec{X}}^{(m)}(w)>: B_{\vec{\alpha} \vec{X}}^{(N-n)}(z) B_{\vec{\beta} \vec{X}}^{(M-m)}(w):
$$

i.e. the OPE coefficients are simply given by the two-point correlators of the lower rank polynomials:

$$
\left.\lambda^{N \mid M}\right|_{m \mid n} \equiv \lambda_{m \mid n}=(z-w)^{n+m}<B_{\vec{\alpha} \vec{X}}^{(n)}(z) B_{\vec{\beta} \vec{X}}^{(m)}(w)>
$$


The last step is to compute the two-point correlators and somehow this again doesn't turn out to be an elementary exercise. Straightforward calculation using the manifest expression (24) for the Bell polynomials and the Wick's theorem leads to complicated sum over partitions which doesn't seem to be realistic to evaluate and doesn't look illuminating or useful for our purposes. Instead, we shall start from the identity

$$
B_{\vec{\alpha} \vec{X}}^{(n)}=\frac{1}{n}\left(\partial B_{\vec{\alpha} \vec{X}}^{(n-1)}+\vec{\alpha} \partial \vec{X} B_{\vec{\alpha} \vec{X}}^{(n-1)}\right)
$$

Inserting this identity in the correlator (62) and using the OPE (59) we obtain the recursion relation

$$
\lambda_{n \mid m}=-\frac{n+m-1}{n} \lambda_{n-1 \mid m}-\frac{\vec{\alpha} \vec{\beta}}{n} \sum_{l=1}^{m-1} \lambda_{n-1 \mid l}
$$

This recursion relation can be simplified by repeating the above procedure and inserting the identity (63) into the correlator $\left\langle B^{(n)} B^{(m-1)}>\right.$, obtaining the similar recursion relation for $\lambda_{n \mid m-1}$ and subtracting it from (64). Then the recursion becomes

$$
n\left(\lambda_{n \mid m}-\lambda_{n \mid m-1}\right)=-(n+m-1) \lambda_{n-1 \mid m}+(n+m-2-\vec{\alpha} \vec{\beta}) \lambda_{n-1 \mid m-1}
$$

with the obvious physical constraints

$$
\lambda_{0 \mid k}=\lambda_{k \mid 0}=\delta_{0 k}
$$

To solve this recursion, define the generating function

$$
F_{\lambda}(x, y)=\sum_{m, n} \lambda_{n \mid m} x^{n} y^{m}
$$

multiply the recursion (65) by $x^{n} y^{m}$ and sum over $m$ and $n$. This leads to the first order partial differential equation for $F_{\lambda}(x, y)$ :

$$
(1-y)(1+x) \partial_{x} F_{\lambda}+y(1-y) \partial_{y} F_{\lambda}+\vec{\alpha} \vec{\beta} y F=0
$$

with the boundary conditions

$$
F_{\lambda}(x, 0)=F_{\lambda}(0, y)=1
$$

This equation isn't hard to solve. Defining

$$
\begin{array}{r}
\xi=\log (1+x), \eta=\log (y) \\
G(x, y)=\log F(x, y)
\end{array}
$$


the equation simplifies according to

$$
\partial_{\xi} G(\xi, \eta)+\partial_{\eta} G(\xi, \eta)-\frac{\vec{\alpha} \vec{\beta} y}{1-e^{-\eta}}=0
$$

and is equivalent to the characteristic ODE system

$$
\begin{aligned}
\frac{d \xi}{d s} & =\frac{d \eta}{d s}=1 \\
\frac{d G}{d s} & =\frac{\vec{\alpha} \vec{\beta} y}{1-e^{-\eta}}
\end{aligned}
$$

so the general solution is

$$
G(\xi, \eta)=H(\xi-\eta)+\vec{\alpha} \vec{\beta} \int \frac{d \eta}{1-e^{-\eta}}
$$

Substituting $G(0, \eta)=0$ then fixes $H$ to be

$$
H(\xi-\eta)=\vec{\alpha} \vec{\beta} \log \left(1-e^{\eta-\xi}\right)
$$

so the solution is

$$
\begin{gathered}
F_{\lambda}(x, y)=\left(\frac{(1+x)(1-y)}{(1+x-y)}\right)^{-\vec{\alpha} \vec{\beta}} \\
\lambda_{n \mid m}=\left.\frac{1}{n ! m !} \partial_{x}^{n} \partial_{y}^{m} F_{\lambda}(x, y)\right|_{x, y=0}
\end{gathered}
$$

This solution, describing the correlator of two Bell polynomial operators is related to the higher-spin algebra in $A d S_{3}$ and determines the parameter $\mu$ of the enveloping $T(\mu)$ of $S U(2)$ 32, 33, 35.

Next, using the OPE (64), (75), it is straightforward to identify the worldsheet correlators of the products of the Bell operators in terms of the $\lambda_{m \mid n}$-numbers, relevant to $w_{\infty}$ and to the $\mathrm{SU}(2)$ enveloping generators, as well as to our SFT ansatz (33)-(35). The result is given by

$$
\begin{array}{r}
<: B_{\vec{\alpha}}^{\left(n_{1}\right)} \ldots B^{\left(n_{p}\right)}:(z): B^{\left(m_{1}\right)} \ldots B^{\left(m_{q}\right)}:(w)>\left.\right|_{N=n_{1}+\ldots+n_{p} ; M=m_{1}+\ldots+m_{q}} \\
=(z-w)^{-N-M} \sum_{\text {partitions }\left[\alpha_{i j}, \beta_{j i}\right]} \prod_{i=1}^{p} \prod_{j=1}^{q}(q !)^{p} \lambda_{\alpha_{i j} \mid \beta_{j i}} \\
\left(\prod_{k=1}^{q} \sigma\left(\alpha_{1 k} \mid \beta_{k 1}\right) !\right)^{-1} \ldots\left(\prod_{k=1}^{q} \sigma\left(\alpha_{p k} \mid \beta_{k p}\right) !\right)^{-1}\left(\prod_{l=1}^{p} \sigma\left(s_{l}\right) !\right)^{-1}
\end{array}
$$


with the constraints

$$
\begin{aligned}
\sum_{j=1}^{q} \alpha_{i j} & =n_{i} \\
\sum_{i=1}^{q} \beta_{j i} & =m_{j} \\
\sum_{i=1}^{p} \alpha_{i j} & =r_{j} \\
\sum_{j=1}^{q} \beta_{j i} & =s_{i} \\
\sum_{j=1}^{q} r_{j}=\sum_{i=1}^{p} n_{i} & =N \\
\sum_{j=1}^{q} m_{j}=\sum_{i=1}^{p} s_{i} & =N
\end{aligned}
$$

where the notations are as follows. We have introduced the exchange numbers $\alpha_{i j}$ indicating how much of the total conformal dimension $n_{i}$ of the $B^{\left(n_{i}\right)}(z)$-operator in the product of the Bell polynomials on the left at $z$ is contributed to its interaction with the operator $B^{\left(m_{j}\right)}(w)$ in the product of the Bell polynomials at $w$ on the right, according to the OPE structure (59). Similarly, the exchange number $\beta_{j i}$ indicates the reduction in the conformal dimension of $B^{\left(m_{j}\right)}(w)$ on the right as a result of its interaction to $B^{\left(n_{i}\right)}(z)$ on the left. Altogether, this corresponds to the order of $(z-w)^{-\alpha_{i j}-\beta_{j i}}$ term in the OPE of these two operators entering the left and the right chains, contributing to the overall correlator. Thus $r_{j}$-numbers, forming the length $q$ partition of $N$ (as opposed to the length $p$ partition of $N$, formed by $n_{i}$ ) indicate the total loss of conformal dimension of the complete operator on the left-hand side at $z$ due to the interaction with the single polynomial $B^{\left(m_{j}\right)}(w)$ on the right. Similarly, the $s_{i}$-numbers, forming the length $p$ partition of $M$ (as opposed to the length $q$ partition of $M$ formed by $m_{i}$ ), indicate the total loss of conformal dimension of the complete operator on the right-hand side at $w$ due to the interaction with the single polynomial $B^{\left(n_{i}\right)}(z)$ on the left. Next, $\sigma\left(\alpha_{j k} \mid \beta_{k j}\right)(j=1, \ldots, p)$ indicates the multiplicity of the array of the exchange numbers $\alpha_{j k} \mid \beta_{k j}$ in $p$ arrays of the length $q$ each: $\left\{\alpha_{j 1}\left|\beta_{1 j}, \ldots, \alpha_{j q}\right| \beta_{q j}\right\}(j=1, \ldots p)$, similarly to (34). Finally, $\sigma\left(s_{l}\right)$ counts multiplicities of the $s$-numbers defined above (again, similarly to (34)) As before, all the partitions are considered ordered. While the sum (76) involving the products of the exchange numbers, 
summed over the partitions, looks tedious, there are some significant simplifications in important cases, when the partitions are summed over.

In fact, we are particularly interested in objects of the type (43) with the partitions summed over, as, e.g. in (33)-(35). Let us again start with the simplest possible warm-up example of summing over the partitions - with all the partition elements summed over uniformly,that is, with the sum being a Bell polynomial of Bell polynomials. Namely, consider the elementary example of a toy string field given by

$$
\Psi=\sum_{M=0}^{\infty} \sum_{q=1}^{M} \sum_{M \mid m_{1} \ldots m_{q}}\left(\prod_{i=1}^{q}\left[\sigma\left(m_{i}\right)\right]^{-1}\right) B^{\left(m_{1}\right)} \ldots B^{\left(m_{q}\right)}
$$

and let's calculate the simplest SFT correlator $\langle\Psi(1) \Psi(0)>$. To calculate this correlator, the expression (76) must be further summed over the partitions according to the definition (78) of the toy $\Psi$. Take the the product (76), defining the correlator $<B^{\left(n_{1}\right)} \ldots . B^{\left(n_{p}\right)}(1) B^{\left(m_{1}\right)} \ldots . B^{\left(m_{q}\right)}(0)>$ and let us begin with the summation over partitions in the second operator at 0 . Consider the first row in the product (76)

$$
\sim \sum_{\text {partitions }\left(n_{1}, s_{1}\right)} \prod_{j=1}^{q}(q !) \lambda_{\alpha_{i j} \mid \beta_{j i}}\left(\prod_{k=1}^{q} \sigma\left(\alpha_{1 k} \mid \beta_{k 1}\right)\right)^{-1}
$$

with the sum taken over the partitions of $n_{1}$ and $s_{1}$ into the exchange number sets. This row completely describes the interaction of $B^{\left(n_{1}\right)}$ with the array of the Bell polynomials at $w$ with $M$ being the total conformal dimension of the array. Let us calculate the effect of the partition summation (78) for this row. Now, in addition to the summation over the above partitions, sum over all the partitions of $M$ with lengths $1 \leq q \leq M$ and uniform weights for each $q$.

It is then straightforward to check that the result will be given by the series expansion coefficient of the following simple generating function:

$$
\begin{array}{r}
\sim \sum_{\text {partitions }\left(n_{1}, s_{1}\right)} \prod_{j=1}^{q}(q !) \lambda_{\alpha_{i j} \mid \beta_{j i}} \\
\left(\prod_{k=1}^{q} \sigma\left(\alpha_{1 k} \mid \beta_{k 1}\right) !\right)^{-1} \\
=\left.\frac{1}{n_{1} ! s_{1} !} \partial_{x}^{n_{1}} \partial_{y}^{s_{1}}\left(\frac{1}{1-F_{\lambda}(x, y)}\right)\right|_{x, y=0}
\end{array}
$$


The same procedure can be repeated for the remaining $p-1$ rows parametrized by $\left(n_{j}, s_{j}\right), j=1, \ldots p$, leading to the

$$
\sim \frac{\partial_{x}^{n_{1}} \partial_{y}^{s_{1}}\left(\frac{1}{1-F_{\lambda}(x, y)}\right) \ldots \partial_{x}^{n_{p}} \partial_{y}^{s_{p}}\left(\frac{1}{1-F_{\lambda}(x, y)}\right)}{n_{1} ! s_{1} ! \ldots n_{p} ! s_{p} !\left[\sigma\left(n_{1} \mid s_{1}\right)\right] ! \ldots\left[\sigma\left(n_{p} \mid s_{p}\right)\right] !}
$$

Finally, let us sum over the partitions for the first string field at 1. For the fixed values of $N$ and $M$ the result is

$$
\left.\sim \frac{1}{M ! N !} \partial_{x}^{N} \partial_{y}^{M}\left(\frac{1}{1-\frac{1}{1-F_{\lambda}}}\right)\right|_{x, y=0}=-\left.\partial_{x}^{N} \partial_{y}^{M} \frac{1}{F_{\lambda}}\right|_{x, y=0},
$$

so the two-point function of the toy string field (78) is

$$
<\Psi(1) \Psi(0)>=\left.e^{-\frac{1}{F_{\lambda}(x, y)}}\right|_{x=y=1}
$$

The objects of the type (78) are of interest to us both because they are relevant to our SFT ansatz and, at the same time, form an operator algebra realization of $w_{\infty}$ and $S U(2)$ envelopings. Namely, instead of the string field $\Psi$ (78) consider the field

$$
\Psi_{N \mid p}=\sum_{q=1}^{p} \sum_{N \mid n_{1} \ldots n_{q}}\left(\prod_{i=1}^{q}\left[\sigma\left(n_{i}\right)\right]^{-1}\right) B^{\left(n_{1}\right)} \ldots B^{\left(n_{q}\right)}
$$

(it is easy to see that the toy string field $\Psi$ is given by $\Psi=\sum_{N=0}^{\infty} \Psi_{N \mid N}$ ) This field is characterized by the numbers $N$ and $p$, with the first being its total conformal dimension and the second indicating the maximum length of the "words" made out of Bell polynomial "letters", contained in the string field "sentence" $\Psi_{N \mid p}$. Let us compute the OPE of two "sentences" $\Psi_{N_{1} \mid p_{1}}(z)$ and $\Psi_{N_{2} \mid p_{2}}(w)$ around the midpoint $\frac{1}{2}(z+w)$. Clearly, the conformal transformation properties of the Bell polynomials imply that the Bell polynomial structure must be preserved under such an operator product. It is also clear, from the OPE structure (61), (76) for the Bell polynomials that the terms ("sentences")of the order $(z-w)^{-N}(N>0)$ would consist of words of conformal dimension $N_{1}+N_{2}-N$ and lengths up to $p_{1}+p_{2}$. For $N=1$ this sends a strong hint towards the emergence of $w_{\infty}$ and of $S U(2)$ envelopings for higher order $N$ values. Indeed, straightforward calculation, using (61) and the recurrence relation (63) leads to the following midpoint OPE simple pole:

$$
\Psi_{N_{1} \mid p_{1}}(z) \Psi_{N_{2} \mid p_{2}}(z)=(z-w)^{-1}\left(N_{2} p_{1}-N_{1} p_{2}\right) \Psi_{N_{1}+N_{2}-1 \mid p_{1}+p_{2}}\left(\frac{z+w}{2}\right)
$$


and the general OPE structure:

$$
\begin{array}{r}
\Psi_{N_{1} \mid p_{1}}(z) \Psi_{N_{2} \mid p_{2}}(z)=(z-w)^{-1}\left(N_{2} p_{1}-N_{1} p_{2}\right) \Psi_{N_{1}+N_{2}-1 \mid p_{1}+p_{2}}\left(\frac{z+w}{2}\right) \\
+\sum_{n=2}^{N_{1}+N_{2}}(z-w)^{-n} \gamma_{n}\left(N_{1}, p_{1} \mid N_{2}, p_{2}\right) \Psi_{N_{1}+N_{2}-n \mid p_{1}+p_{2}}\left(\frac{z+w}{2}\right)
\end{array}
$$

Although we have not computed the $\gamma_{n}$ coefficients in this paper explicitly, such a computation doesn't look a conceptual challenge and the result must be anyway determined by combinations of the $\lambda$-numbers (75) stemming from the two-point correlators of the Bell polynomials. So we recognize classical $w_{\infty}$ at the simple pole and the enveloping $T(\mu)$ of $\mathrm{SU}(2)$ at the higher order singularities with the $\mu$-parameter related to the $\lambda$-numbers. Note that this is the midpoint OPE. If, for example, one needs to compute the OPE around the $w$-point, the right-hand side of (85) must be shifted from $\frac{1}{2}(z+w)$ to $w$ by the appropriate series expansion in $\frac{1}{2}(z-w)$. This way, the full enveloping algebra will appear, for example, in commutators of the charges $\oint d z \Psi_{N \mid n}$.

Now consider a more general example of a string field, relevant to our ansatz. Consider again a generating function of the normalized Bell polynomials:

$$
H(B)=\sum_{n=0}^{\infty} \frac{h_{n}}{n !} B_{\vec{\alpha} \vec{X}}^{(n)}
$$

and the string field given by

$$
\Psi=G \circ H(B)
$$

where

$$
G(x)=\sum_{n=0}^{\infty} \frac{g_{n}}{n !} x^{n}
$$

with some fixed coefficients $h_{n}$ and $g_{n}$. Using the OPE rules and the formalism developed above, the calculation of the two-point function gives:

$$
\begin{array}{r}
<\Psi(1) \Psi(0)>= \\
\sum_{N=0}^{\infty} \sum_{M=0}^{\infty} \sum_{n=0}^{N} \sum_{m=0}^{M} g_{n} g_{m} \sum_{N \mid p_{1} \ldots p_{n}} \sum_{M \mid q_{1} \ldots q_{m}} \frac{h_{p_{1}} \ldots h_{p_{n}} h_{q_{1}} \ldots h_{q_{m}}}{p_{1} ! \ldots p_{n} ! q_{1} ! \ldots q_{m} ! \prod_{i, j}\left[\sigma\left(p_{i}\right)\right] !\left[\sigma\left(q_{j}\right)\right] !} \\
\times \sum_{\text {partitions }\left[\alpha_{i j}, \beta_{j i}\right]} \prod_{i=1}^{n} \prod_{j=1}^{m} \lambda_{\alpha_{i j} \mid \beta_{j i}}\left(\prod_{k=1}^{m} \sigma\left(\alpha_{1 k} \mid \beta_{k 1}\right) !\right)^{-1} \ldots\left(\prod_{k=1}^{m} \sigma\left(\alpha_{n k} \mid \beta_{k n}\right) !\right)^{-1}\left(\prod_{l=1}^{n} \sigma\left(s_{l}\right) !\right)^{-1}
\end{array}
$$


with the constraints

$$
\begin{gathered}
\sum_{j=1}^{m} \alpha_{i j}=n_{i} ; \sum_{i=1}^{n} \beta_{j i}=m_{j} \\
\sum_{i=1}^{n} \alpha_{i j}=r_{j} ; \sum_{j=1}^{m} \beta_{j i}=s_{i} \\
\sum_{j=1}^{m} r_{j}=\sum_{i=1}^{n} n_{i}=N \\
\sum_{j=1}^{m} m_{j}=\sum_{i=1}^{n} s_{i}=N
\end{gathered}
$$

with the multiplicity $\sigma$-factors, exchange numbers and $r, s$-numbers defined as before. By direct comparison, it is straightforward to realize that the lengthy expression on the righthand side of (86), (87) is just a series expansion of the relatively simple generating composite function, that is, it can be cast as simply as

$$
<\Psi(1) \Psi(0)>=\left.\tilde{G}\left(H\left(F_{\lambda}(x, y)\right) ; H\left(F_{\lambda}(x, y)\right)\right)\right|_{x=y=1}
$$

where the function of two variables $\tilde{G}(x, y)$ is related to the function $G(x)$ with the single argument according to

$$
\tilde{G}(x, y)=\sum_{m, n} \frac{g_{m} g_{n}}{m ! n !} x^{m} y^{n}
$$

where $g_{n}$ are the expansion coefficients of $G$. It is instructive to generalize this two-point correlator to the case of two different string fields, that is, for the case of string fields of the type (84) with the different $H$-functions, but with the same $G$-function. The calculation, completely similar to the above, gives:

$$
\begin{array}{r}
\Psi_{1}=G \circ H_{1}(B) \\
\Psi_{2}=G \circ H_{2}(B) \\
<\Psi_{1}(1) \Psi_{2}(0)>=\left.\tilde{G}\left(H_{1}\left(F_{\lambda}(x, y)\right) ; H_{2}\left(F_{\lambda}(x, y)\right)\right)\right|_{x=y=1}
\end{array}
$$

The next step in the computation the SFT correlators relevant to the equations of motion in SFT is to determine how the global conformal transformations by $I(z)$ and $g_{k}(z) \equiv g \circ f_{k}^{3}(z)$ act on the string fields of the type (84). Using the transformations (48)-(58), it is not difficult to deduce that, under any of these conformal transformations (denoted by $f(z)$ 
for the brevity) the string field (84) transforms as

$$
\begin{array}{r}
\Psi \equiv G(H(B)) \rightarrow \hat{f} \Psi \\
=\frac{d G}{d H} \sum_{n=1}^{\infty} \sum_{k=1}^{n} \alpha_{f}(k, n)\left[(B-1) \partial_{B}^{k} H(B)+\partial_{B}^{k-1} H(B)\right] \\
+\frac{d^{2} G}{d H^{2}} \sum_{m, n=1 ; m<n}^{\infty} \sum_{k, l=1 ; m<n}^{k+l=n-1} \partial^{k} H \partial^{l} H \beta_{f}(k, l \mid n)
\end{array}
$$

with the differentiation rules for $G$ and $H$ explained above (31), (32) and with the coeffi-

cients $\alpha_{f}$ and $\beta_{f}$ related to the conformal transformations by $I(z)=-\frac{1}{z}$ and $g \circ f_{k}^{3}(z)(5)$,

(49) according to:

$$
\begin{array}{r}
\alpha_{I(z)}(k, n)=\frac{1}{k !} \frac{\Gamma(2 n-1)}{\Gamma(2 n-k)} \\
\alpha_{g \circ f_{1}^{3}(z)}(k, n)=\frac{1}{k}\left(-\frac{2}{3}\right)^{n-k} \sum_{a, b, c \mid a+b+c=k-1} \frac{e^{\frac{i \pi}{2}(a-b)} 2^{-c}}{a ! b ! c !} \\
\times \frac{\Gamma\left(1-\frac{5}{3}(1-n)\right) \Gamma\left(1-\frac{1}{3}(1-n)\right) \Gamma(1-2(1-n))}{\Gamma\left(1-\frac{5}{3}(1-n)-a\right) \Gamma\left(1-\frac{1}{3}(1-n)-b\right) \Gamma(1-2(1-n)-c)} \\
\alpha_{g \circ f_{2}^{3}(z)}(k, n)=\frac{1}{k}\left(-\frac{8}{3}\right)^{n-k} \quad \sum_{a, b, c \mid a+b+c=k-1} \frac{e^{\left(\frac{i \pi}{2}(a-b)+\frac{2 i \pi c}{3}\right)} 2^{-c}}{a ! b ! c !} \\
\times \frac{\Gamma\left(1-\frac{5}{3}(1-n)\right) \Gamma\left(1-\frac{1}{3}(1-n)\right) \Gamma(1-2(1-n))}{\Gamma\left(1-\frac{5}{3}(1-n)-a\right) \Gamma\left(1-\frac{1}{3}(1-n)-b\right) \Gamma(1-2(1-n)-c)} \\
\alpha_{g \circ f_{3}^{3}(z)}(k, n)=\frac{1}{k}\left(\frac{8}{3}\right)^{n-k} \quad \sum_{a, b, c \mid a+b+c=k-1} \frac{e^{\left(\frac{i \pi}{2}(a-b)-\frac{2 i \pi c}{3}\right)} 2^{-c}}{a ! b ! c !} \\
\times \frac{\Gamma\left(1-\frac{5}{3}(1-n)\right) \Gamma\left(1-\frac{1}{3}(1-n)\right) \Gamma(1-2(1-n))}{\Gamma\left(1-\frac{5}{3}(1-n)-a\right) \Gamma\left(1-\frac{1}{3}(1-n)-b\right) \Gamma(1-2(1-n)-c)}
\end{array}
$$


and

$$
\begin{aligned}
& \beta_{I(z)}(k, l \mid n)=\frac{2}{(k+l-1) !}\left(-\frac{2}{3}\right)^{n-k-l+1} \frac{\Gamma(-3)}{\Gamma(-1-k-l)} \\
& \beta_{g \circ f_{1}^{3}(z)}(k, l \mid n)=\frac{1}{k+l-1}\left(-\frac{8}{3}\right)^{n-k-l+1} \\
& \times \sum_{a, b, c \mid a+b+c=k+l-2} \frac{e^{\frac{i \pi}{2}(a-b)} 2^{-c}}{a ! b ! c !} \frac{\Gamma\left(-\frac{7}{3}\right) \Gamma\left(\frac{1}{3}\right) \Gamma(-3)}{\Gamma\left(-\frac{7}{3}-a\right) \Gamma\left(\frac{1}{3}-b\right) \Gamma(-3-c)} \\
& \beta_{g \circ f_{2}^{3}(z)}(k, l \mid n)=\frac{1}{k+l-1}\left(-\frac{8}{3}\right)^{n-k-l+1} \\
& \times \sum_{a, b, c \mid a+b+c=k+l-2} \frac{e^{\left(\frac{i \pi}{2}(a-b)+\frac{2 i \pi c}{3}\right)} 2^{-c}}{a ! b ! c !} \frac{\Gamma\left(-\frac{7}{3}\right) \Gamma\left(\frac{1}{3}\right) \Gamma(-3)}{\Gamma\left(-\frac{7}{3}-a\right) \Gamma\left(\frac{1}{3}-b\right) \Gamma(-3-c)} \\
& \beta_{g \circ f_{2}^{3}(z)}(k, l \mid n)=\frac{1}{k+l-1}\left(\frac{8}{3}\right)^{n-k-l+1} \\
& \times \sum_{a, b, c \mid a+b+c=k+l-2} \frac{e^{\left(\frac{i \pi}{2}(a-b)-\frac{2 i \pi c}{3}\right)} 2^{-c}}{a ! b ! c !} \frac{\Gamma\left(-\frac{7}{3}\right) \Gamma\left(\frac{1}{3}\right) \Gamma(-3)}{\Gamma\left(-\frac{7}{3}-a\right) \Gamma\left(\frac{1}{3}-b\right) \Gamma(-3-c)}
\end{aligned}
$$

Furthermore, our notations in (91) are defined as follows. Consider a function of the normalized Bell polynomials $f\left(B_{1}, B_{2}, \ldots.\right)=\sum_{n>0} f_{n} B_{\vec{\psi}}^{(n)}$ and the associate function $f(B)$ given by the formal series in auxiliary argument $B f(B)=\sum_{n>0} f_{n} B^{n}$ Consider a transformation $f(B) \rightarrow g(B)$ where $g(B)=\sum_{n} g_{n} B^{n}$ can obrained from $f$ by differentiation over $B$, integration, multiplication(s) by $B$ and/or their combination. Then the formal series for $g(B)$ define the new associate generating function of the normalized Bell polynomials $g\left(B_{1}, \ldots, B_{n}\right)=\sum_{n} g_{n} B_{\vec{\psi}}^{(n)}$ by identifying $B^{n} \rightarrow B_{\vec{\psi}}^{(n)}$.

This fully determines the transformations of the SFT string field ansatz under the conformal transformations mapping the worlsheets to the wedges of the single disc and then to the single half-plane. The next step is to point out the action of the BRST charge on the string field ansatz. This too can be reduced to the transformations of the ansatz functions $G$ and $H$. Since the only SFT correlator involving the BRST charge is $<<Q \Psi \mid \Psi>>=<Q \Psi(0) I \circ \Psi(0)>$ and both $\Psi$ and $I \circ \Psi$ are proportional to $c$, the only terms in the commutator with the BRST charge (12) contributing to this correlator are those proportional to $\partial c c$ and $\partial^{2} c c$, while all the terms in $Q \Psi$ containing higher derivatives of the $c$-ghost don't contribute to the correlator since $\partial^{n} c c \sim B_{\sigma}^{(n-1)} \partial c c$ with $\sigma$ being the bosonized $c$-ghost. Such terms do not contribute to the two-point correlators since the Bell polynomials in the derivatives of the bosonized $c$-ghost: $B_{\sigma}^{(n-1)}$ cannot fully contract to the $c$-ghost of the opposite string field for $n>2$. Using the OPE (61), (62), (75) it 
is straightforward to show that for the string field $\Psi$ (33)-(35) the relevant terms in the BRST transformation are given by:

$$
\begin{array}{r}
Q \Psi \equiv Q c G(H(B))=\partial c c\left(B \partial_{B} G(H(B))-G(H(B))\right) \\
+\frac{1}{2} \partial^{2} c c B \partial_{B}^{2} G(H(B)) \\
=\partial c\left(B \partial_{B} \Psi-\Psi\right)+\frac{1}{2} \partial^{2} c B \partial_{B}^{2} \Psi
\end{array}
$$

with the notations explained above. With all the above identities it is now straightforward to calculate the SFT correlators. The three-point correlator is then computed to give

$$
\begin{array}{r}
<<g_{1} \circ G(H(B))(0) g_{2} \circ G(H(B))(0) g_{3} \circ G(H(B))(0)>> \\
\equiv<<\Psi \mid \Psi \star \Psi>>=T_{1}+T_{2}+T_{3}+T_{4}
\end{array}
$$

Here

$$
\begin{aligned}
& T_{1}=\sum_{n_{1}, n_{2}, n_{3}=0}^{\infty} \sum_{m_{1}, m_{2}, m_{3}=0}^{\infty} \sum_{q_{1}, q_{2}, q_{3}=0}^{\infty} \sum_{k_{1}=0}^{m_{1}} \sum_{k_{2}=0}^{m_{2}} \sum_{k_{3}=0}^{m_{3}} \sum_{N, R, T=0}^{\infty} \\
& \sum_{N \mid n_{1} \ldots n_{q_{1}}} \sum_{R \mid r_{1} \ldots r_{q_{2}}} \sum_{T \mid t_{1} \ldots t_{q_{3}}} \sum_{N_{1}=0}^{N+m_{1}-k_{1}} \sum_{R_{1}=0}^{R+m_{2}} \sum_{T_{1}=0}^{T+k_{3}-k_{3}} \\
& \left\{\frac{g_{q_{1}+1} g_{q_{2}+1} g_{q_{3}+1}}{q_{1} ! q_{2} ! q_{3} !} h_{m_{1}} h_{m_{2}} h_{m_{3}} h_{n_{1}} \ldots . h_{n_{q_{1}}} h_{r_{1}} \ldots h_{r_{q_{2}}} h_{t_{1}} \ldots . h_{t_{q_{3}}}\right. \\
& \times \prod_{i_{1}=1}^{q_{2}+1} \prod_{j_{1}=1}^{q_{1}+1} \prod_{i_{2}=1}^{q_{3}+1} \prod_{j_{2}=1}^{q_{1}+1} \prod_{i_{3}=1}^{q_{3}+1} \prod_{j_{3}=1}^{q_{2}+1} \lambda_{\alpha_{i_{1} j_{1}} \mid \beta_{j_{1} i_{1}}} \lambda_{\tilde{\alpha}_{i_{2} j_{2}} \mid \beta_{j_{2} i_{2}}} \lambda_{\tilde{\alpha}_{i_{2} j_{2}} \mid \tilde{\beta}_{j_{2} i_{2}}} \\
& \prod_{\mu=1}^{q_{1}+1} \sigma^{-1}\left(\lambda_{\alpha_{\mu, 1} \mid \beta_{1, \mu}}, \ldots \lambda_{\alpha_{\mu, q_{2}+1} \mid \beta_{q_{2}+1, \mu}}\right) \sigma^{-1}\left(\lambda_{\tilde{\alpha}_{\mu, 1} \mid \beta_{1, \mu}}, \ldots \lambda_{\tilde{\alpha}_{\mu, q_{3}+1} \mid \beta_{q_{3}+1, \mu}}\right) \\
& \prod_{\nu=1}^{q_{2}+1} \sigma^{-1}\left(\lambda_{\tilde{\alpha}_{\mu, 1} \mid \tilde{\beta}_{1, \mu}}, \ldots \lambda_{\tilde{\alpha}_{\nu, q_{3}+1} \mid \tilde{\beta}_{q_{3}+1, \nu}}\right) \\
& \times \sigma^{-1}\left(s_{1}^{(1)}, \ldots s_{q_{2}+1}^{(1)}\right) \sigma^{-1}\left(\tilde{s}_{1}^{(1)}, \ldots \tilde{s}_{q_{3}+1}^{(1)}\right) \sigma^{-1}\left(s_{1}^{(2)}, \ldots s_{q_{1}+1}^{(2)}\right) \sigma^{-1}\left(\tilde{s}_{1}^{(2)}, \ldots \tilde{s}_{q_{3}+1}^{(2)}\right) \sigma^{-1}\left(s_{1}^{(3)}, \ldots s_{q_{1}+1}^{(3)}\right) \\
& \sigma^{-1}\left(\tilde{s}_{1}^{(3)}, \ldots \tilde{s}_{q_{2}+1}^{(3)}\right)\left(\left(q_{2}+1\right) !\left(q_{3}+1\right) !\right)^{q_{1}+1}\left(\left(q_{3}+1\right) !\right)^{q_{2}+1} \\
& \left.\times 2^{N+T_{1}-N_{1}+m_{1}-k_{1}}(\sqrt{3})^{N+R+T+m_{1}-k_{1}+m_{2}-k_{2}+m_{3}-k_{3}}\right\} \\
& + \text { permutations }\left(g_{1} \circ \Psi, g_{2} \circ \Psi, g_{3} \circ \Psi\right)
\end{aligned}
$$

where the exchange numbers are defined similarly to the previous case, as well as the $\sigma^{-1}$ factors, defined by products of array multiplicities in the relevant partitions. Next, the 
$s, \tilde{s}$-numbers are similar to the $r, s$-numbers defined previously and are related to conformal dimension losses of string field components due to interactions with partition elements (individual Bell polynomials) in components of two opposite string fields. Altogether , these numbers satisfy the following constraints:

$$
\begin{gathered}
\sum_{j=1}^{q_{2}+1} \alpha_{i j}+\sum_{j=1}^{q_{3}+1} \tilde{\alpha}_{i j}=n_{i}\left(i=1, \ldots, q_{1}+1\right) \\
\sum_{j=1}^{q_{1}+1} \beta_{i j}+\sum_{j=1}^{q_{3}+1} \tilde{\beta}_{i j}=r_{i}\left(i=1, \ldots, q_{2}+1\right) \\
\sum_{j=1}^{q_{1}+1} \gamma_{i j}+\sum_{j=1}^{q_{2}+1} \tilde{\gamma}_{i j}=t_{i}\left(i=1, \ldots, q_{3}+1\right)
\end{gathered}
$$

and

$$
\begin{aligned}
& \sum_{i=1}^{q_{1}+1} \alpha_{i j}=s_{j}^{(1)}\left(j=1, \ldots, q_{2}+1\right) \\
& \sum_{i=1}^{q_{1}+1} \tilde{\alpha}_{i j}=\tilde{s}_{j}^{(1)}\left(j=1, \ldots, q_{3}+1\right) \\
& \sum_{i=1}^{q_{2}+1} \alpha_{i j}=s_{j}^{(2)}\left(j=1, \ldots, q_{1}+1\right) \\
& \sum_{i=1}^{q_{2}+1} \tilde{\beta}_{i j}=\tilde{s}_{j}^{(2)}\left(j=1, \ldots, q_{3}+1\right) \\
& \sum_{i=1}^{q_{3}+1} \gamma_{i j}=s_{j}^{(3)}\left(j=1, \ldots, q_{1}+1\right) \\
& \sum_{i=1}^{q_{2}+1} \tilde{\gamma}_{i j}=\tilde{s}_{j}^{(3)}\left(j=1, \ldots, q_{2}+1\right)
\end{aligned}
$$

and furthermore

$$
\begin{aligned}
N+m_{1}-k_{1} & =\sum_{i=1}^{q_{1}+1} n_{i}=\sum_{j=1}^{q_{2}+1} s_{j}^{(1)}+\sum_{j=1}^{q_{3}+1} \tilde{s}_{j}^{(1)} \\
R+m_{2}-k_{2} & =\sum_{i=1}^{q_{2}+1} r_{i}=\sum_{j=1}^{q_{1}+1} s_{j}^{(2)}+\sum_{j=1}^{q_{3}+1} \tilde{s}_{j}^{(2)} \\
T+m_{3}-k_{3} & =\sum_{i=1}^{q_{3}+1} t_{i}=\sum_{j=1}^{q_{1}+1} s_{j}^{(3)}+\sum_{j=1}^{q_{2}+1} \tilde{s}_{j}^{(3)}
\end{aligned}
$$


In other words, the exchange numbers, that form the OPE structure of the Bell polynomial products, can be visualized as "partitions of partitions" of the conformal dimensions of the string field components.

This constitutes $T_{1}$, the first out of 4 terms contributing to the 3 -point correlator. The remaining three can be obtained from $T_{1}$ by few simple replacements/manipulations. That is, $T_{2}$ is obtained from $T_{1}$ by replacing one of three $\alpha$ coefficients in (96) by the $\beta$-coefficient: $\alpha\left(k_{1}, n_{1}\right) \rightarrow \beta\left(k_{1}, l_{1} \mid n_{1}\right)$, with $k_{1}, l_{1}$ being summed over from 0 to $k_{1}+l_{1}=n_{1}$; inserting an extra h-coefficient in the sum according to: $h_{m_{1}} h_{m_{2}} h_{m_{3}} \rightarrow h_{m_{1}} h_{m_{2}} h_{m_{3}} h_{m_{4}}$, replacing the difference $m_{1}-k_{1} \rightarrow m_{1}+m_{4}-k_{1}-l_{1}$ and finally replacing $q_{1}+1 \rightarrow q_{1}+2$ in the upper limits in the products over $j_{1}$ and $j_{2}$ in (96)-(99), as well as in the relevant $g$-coefficient (the first among three in (96)) and in the relevant $\sigma^{-1}$-factors, increasing their number of arguments by one unit - and finally, permuting over the conformal transformations by $g_{1}$, $g_{2}, g_{3}$, as in $T_{1}$. Thus the $T_{1}$-contribution has the $\alpha \alpha \alpha$-structure, while $T_{2}$ carries the $\beta \alpha \alpha$ structure. Similarly, to obtain $T_{3}$ out of $T_{2}$, one further replaces the second $\alpha$-coefficient by the $\beta$-coefficient: $\alpha\left(k_{2}, n_{2}\right) \rightarrow \beta\left(k_{2}, l_{2} \mid n_{2}\right)$, inserts an extra $h$-coefficient in the product: $h_{m_{1}} \ldots h_{m_{4}} \rightarrow h_{m_{1}} \ldots h_{m_{4}} h_{m_{5}}$, and further replaces $m_{2}-k_{2} \rightarrow m_{2}+m_{5}-k_{2}-l_{2}$ and $q_{2}+1 \rightarrow q_{2}+2$ according to the prescriptions explained above. This, upon the permutation over the conformal transformations, similar to the above, gives the $T_{3}$-contribution with the $\beta \beta \alpha$-structure. The final contribution, $T_{4}$, having the $\beta \beta \beta$-structure, is obtained similarly from $T_{3}$ by replacing the last remaining $\alpha$ with $\beta$ and performing the manipulations identical to those described above. The overall expression for the three-point correlator thus looks complex enough. Nevertheless, it is straightforward to check that, just as in the elementary warm-up example demonstrated previously, the complicated sum given by (96)-(99) can be converted successfully into the generating composite function and identified with its series expansion. Namely, we obtain:

$$
\begin{array}{r}
<<\Psi \mid \Psi \star \Psi>>\equiv<<g_{1} \circ G(H(B))(0) g_{2} \circ G(H(B))(0) g_{3} \circ G(H(B))(0)>> \\
=\sum_{j=1}^{4} K_{j}\left(G\left(H\left(F_{\lambda}\right)\right)\right)
\end{array}
$$


where

$$
\begin{aligned}
& K_{1}\left(G\left(H\left(F_{\lambda}\right)\right)\right)=\sum_{n_{1}=0}^{\infty} \sum_{n_{2}=0}^{\infty} \sum_{n_{3}=0}^{\infty} \sum_{k_{1}=0}^{n_{1}} \sum_{k_{2}=0}^{n_{2}} \sum_{k_{3}=0}^{n_{3}} \sum_{Q=1}^{k_{1}} \sum_{R=1}^{k_{2}} \sum_{S=1}^{k_{3}} \\
& \left\{\left[\alpha_{g_{1}}\left(k_{1}, n_{1}\right) \alpha_{g_{2}}\left(k_{2}, n_{2}\right) \alpha_{g_{3}}\left(k_{3}, n_{3}\right)+\alpha_{g_{1}}\left(k_{1}, n_{1}\right) \alpha_{g_{3}}\left(k_{2}, n_{2}\right) \alpha_{g_{2}}\left(k_{3}, n_{3}\right)\right.\right. \\
& +\alpha_{g_{2}}\left(k_{1}, n_{1}\right) \alpha_{g_{1}}\left(k_{2}, n_{2}\right) \alpha_{g_{3}}\left(k_{3}, n_{3}\right)+\alpha_{g_{2}}\left(k_{1}, n_{1}\right) \alpha_{g_{3}}\left(k_{2}, n_{2}\right) \alpha_{g_{1}}\left(k_{3}, n_{3}\right) \\
& \left.+\alpha_{g_{3}}\left(k_{1}, n_{1}\right) \alpha_{g_{2}}\left(k_{2}, n_{2}\right) \alpha_{g_{1}}\left(k_{3}, n_{3}\right)+\alpha_{g_{3}}\left(k_{1}, n_{1}\right) \alpha_{g_{1}}\left(k_{2}, n_{2}\right) \alpha_{g_{2}}\left(k_{3}, n_{3}\right)\right\} \\
& \times\left.\left.\left.\partial_{F_{\lambda}}^{k_{1}} H\left(F_{\lambda}(x, y)\right)\right|_{x, y=\sqrt{3}} \partial_{F_{\lambda}}^{k_{2}} H\left(F_{\lambda}(x, y)\right)\right|_{x, y=2 \sqrt{3}} \partial_{F_{\lambda}}^{k_{3}} H\left(F_{\lambda}(x, y)\right)\right|_{x, y=\sqrt{3}} \\
& \times\left.\left.\left. G^{\prime}\left(\partial^{Q} H\left(F_{\lambda}(x, y)\right)\right)\right|_{x, y=\sqrt{3}} G^{\prime}\left(\partial^{R} H\left(F_{\lambda}(x, y)\right)\right)\right|_{x, y=2 \sqrt{3}} G^{\prime}\left(\partial^{S} H\left(F_{\lambda}(x, y)\right)\right)\right|_{x, y=\sqrt{3}}
\end{aligned}
$$

$$
\begin{array}{r}
K_{2}\left(G\left(H\left(F_{\lambda}\right)\right)\right)=\sum_{n_{1}=0}^{\infty} \sum_{n_{2}=0}^{\infty} \sum_{n_{3}=0}^{\infty} \sum_{k_{1}, l_{1}=0}^{k_{1}+l_{1}=n_{1}} \sum_{k_{2}=0}^{n_{2}} \sum_{k_{3}=0}^{n_{3}} \sum_{Q=1}^{k_{1}+l_{1}-1} \sum_{R=1}^{k_{2}} \sum_{S=1}^{k_{3}} \\
\beta_{g_{1}}\left(k_{1}, l_{1} \mid n_{1}\right)\left(\alpha_{g_{2}}\left(k_{2}, n_{2}\right) \alpha_{g_{3}}\left(k_{3}, n_{3}\right)+\alpha_{g_{3}}\left(k_{2}, n_{2}\right) \alpha_{g_{2}}\left(k_{3}, n_{3}\right)\right)
\end{array}
$$$$
\times\left.\left.\left. G^{\prime \prime}\left(\partial^{Q} H\left(F_{\lambda}(x, y)\right)\right)\right|_{x, y=\sqrt{3}} G^{\prime}\left(\partial^{R} H\left(F_{\lambda}(x, y)\right)\right)\right|_{x, y=2 \sqrt{3}} G^{\prime}\left(\partial^{S} H\left(F_{\lambda}(x, y)\right)\right)\right|_{x, y=\sqrt{3}}
$$$$
\times\left(\left.\left.\partial_{F_{\lambda}}^{k_{1}} H\left(F_{\lambda}(x, y)\right)\right|_{x, y=\sqrt{3}} \partial_{F_{\lambda}}^{l_{1}} H\left(F_{\lambda}(x, y)\right)\right|_{x, y=\sqrt{3}}\right.
$$$$
\left.\times\left.\left.\partial_{F_{\lambda}}^{k_{2}} H\left(F_{\lambda}(x, y)\right)\right|_{x, y=2 \sqrt{3}} \partial_{F_{\lambda}}^{k_{3}} H\left(F_{\lambda}(x, y)\right)\right|_{x, y=\sqrt{3}}\right)
$$$$
+\beta_{g_{2}}\left(k_{1}, l_{1} \mid n_{1}\right)\left(\alpha_{g_{1}}\left(k_{2}, n_{2}\right) \alpha_{g_{3}}\left(k_{3}, n_{3}\right)+\alpha_{g_{3}}\left(k_{2}, n_{2}\right) \alpha_{g_{1}}\left(k_{3}, n_{3}\right)\right)
$$$$
\times\left.\left.\left. G^{\prime \prime}\left(\partial^{Q} H\left(F_{\lambda}(x, y)\right)\right)\right|_{x, y=2 \sqrt{3}} G^{\prime}\left(\partial^{R} H\left(F_{\lambda}(x, y)\right)\right)\right|_{x, y=\sqrt{3}} G^{\prime}\left(\partial^{S} H\left(F_{\lambda}(x, y)\right)\right)\right|_{x, y=\sqrt{3}}
$$

$$
\begin{aligned}
& \times\left.\left.\partial_{F_{\lambda}}^{k_{1}} H\left(F_{\lambda}(x, y)\right)\right|_{x, y=2 \sqrt{3}} \partial_{F_{\lambda}}^{l_{1}} H\left(F_{\lambda}(x, y)\right)\right|_{x, y=2 \sqrt{3}} \\
& \times\left.\left.\left.\partial_{F_{\lambda}}^{k_{2}} H\left(F_{\lambda}(x, y)\right)\right|_{x, y=\sqrt{3}} \partial_{F_{\lambda}}^{k_{3}} H\left(F_{\lambda}(x, y)\right)\right|_{x, y=\sqrt{3}}\right) \\
&+\beta_{g_{3}}\left(k_{1}, l_{1} \mid n_{1}\right)\left(\alpha_{g_{1}}\left(k_{2}, n_{2}\right) \alpha_{g_{2}}\left(k_{3}, n_{3}\right)+\alpha_{g_{2}}\left(k_{2}, n_{2}\right) \alpha_{g_{1}}\left(k_{3}, n_{3}\right)\right) \\
& \times\left.\left.\left. G^{\prime}\left(\partial^{Q} H\left(F_{\lambda}(x, y)\right)\right)\right|_{x, y=\sqrt{3}} G^{\prime}\left(\partial^{R} H\left(F_{\lambda}(x, y)\right)\right)\right|_{x, y=\sqrt{3}} G^{\prime}\left(\partial^{S} H\left(F_{\lambda}(x, y)\right)\right)\right|_{x, y=2 \sqrt{3}} \\
& \times\left(\left.\left.\partial_{F_{\lambda}}^{k_{1}} H\left(F_{\lambda}(x, y)\right)\right|_{x, y=\sqrt{3}} \partial_{F_{\lambda}}^{l_{1}} H\left(F_{\lambda}(x, y)\right)\right|_{x, y=\sqrt{3}}\right. \\
&\left.\times\left.\left.\partial_{F_{\lambda}}^{k_{2}} H\left(F_{\lambda}(x, y)\right)\right|_{x, y=\sqrt{3}} \partial_{F_{\lambda}}^{k_{3}} H\left(F_{\lambda}(x, y)\right)\right|_{x, y=2 \sqrt{3}}\right)
\end{aligned}
$$




$$
\begin{aligned}
& K_{3}\left(G\left(H\left(F_{\lambda}\right)\right)\right)=\sum_{n_{1}=0}^{\infty} \sum_{n_{2}=0}^{\infty} \sum_{n_{3}=0}^{\infty} \sum_{k_{1}, l_{1}=0 ; k_{1}<l_{1}}^{k_{1}+l_{1}=n_{1}} \sum_{k_{2}, l_{2}=0 ; k_{2}<l_{2}}^{k_{2}+l_{2}=n_{2}} \sum_{k_{3}=0}^{n_{3}} \sum_{Q=1}^{k_{1}+l_{1}-1} \sum_{R=1}^{k_{2}+l_{2}-1} \sum_{S=1}^{k_{3}} \\
& \left(\beta_{g_{1}}\left(k_{1}, l_{1} \mid n_{1}\right) \beta_{g_{2}}\left(k_{2}, l_{2} \mid n_{2}\right)+\beta_{g_{2}}\left(k_{1}, l_{1} \mid n_{1}\right) \beta_{g_{1}}\left(k_{2},, l_{2} \mid n_{2}\right)\right) \alpha_{g_{3}}\left(k_{3}, n_{3}\right) \\
& \times\left.\left.\left. G^{\prime \prime}\left(\partial^{Q} H\left(F_{\lambda}(x, y)\right)\right)\right|_{x, y=\sqrt{3}} G^{\prime \prime}\left(\partial^{R} H\left(F_{\lambda}(x, y)\right)\right)\right|_{x, y=2 \sqrt{3}} G^{\prime}\left(\partial^{S} H\left(F_{\lambda}(x, y)\right)\right)\right|_{x, y=\sqrt{3}} \\
& \times\left(\left.\left.\left.\partial_{F_{\lambda}}^{k_{1}} H\left(F_{\lambda}(x, y)\right)\right|_{x, y=\sqrt{3}} \partial_{F_{\lambda}}^{l_{1}} H\left(F_{\lambda}(x, y)\right)\right|_{x, y=\sqrt{3}} \partial_{F_{\lambda}}^{k_{2}} H\left(F_{\lambda}(x, y)\right)\right|_{x, y=2 \sqrt{3}}\right. \\
& \left.\times\left.\partial_{F_{\lambda}}^{l_{2}} H\left(F_{\lambda}(x, y)\right)\right|_{x, y=2 \sqrt{3}}\right)\left.\partial_{F_{\lambda}}^{k_{3}} H\left(F_{\lambda}(x, y)\right)\right|_{x, y=\sqrt{3}} \\
& +\left(\beta_{g_{1}}\left(k_{1}, l_{1} \mid n_{1}\right) \beta_{g_{3}}\left(k_{2}, l_{2} \mid n_{2}\right)+\beta_{g_{3}}\left(k_{1}, l_{1} \mid n_{1}\right) \beta_{g_{1}}\left(k_{2},, l_{2} \mid n_{2}\right)\right) \alpha_{g_{2}}\left(k_{3}, n_{3}\right) \\
& \times\left.\left.\left. G^{\prime \prime}\left(\partial^{Q} H\left(F_{\lambda}(x, y)\right)\right)\right|_{x, y=2 \sqrt{3}} G^{\prime \prime}\left(\partial^{R} H\left(F_{\lambda}(x, y)\right)\right)\right|_{x, y=\sqrt{3}} G^{\prime}\left(\partial^{S} H\left(F_{\lambda}(x, y)\right)\right)\right|_{x, y=\sqrt{3}} \\
& \times\left(\left.\left.\left.\partial_{F_{\lambda}}^{k_{1}} H\left(F_{\lambda}(x, y)\right)\right|_{x, y=2 \sqrt{3}} \partial_{F_{\lambda}}^{l_{1}} H\left(F_{\lambda}(x, y)\right)\right|_{x, y=2 \sqrt{3}} \partial_{F_{\lambda}}^{k_{2}} H\left(F_{\lambda}(x, y)\right)\right|_{x, y=\sqrt{3}}\right. \\
& \left.\times\left.\partial_{F_{\lambda}}^{l_{2}} H\left(F_{\lambda}(x, y)\right)\right|_{x, y=\sqrt{3}}\right)\left.\partial_{F_{\lambda}}^{k_{3}} H\left(F_{\lambda}(x, y)\right)\right|_{x, y=\sqrt{3}} \\
& +\left(\beta_{g_{2}}\left(k_{1}, l_{1} \mid n_{1}\right) \beta_{g_{3}}\left(k_{2},, l_{2} \mid n_{2}\right)+\beta_{g_{3}}\left(k_{1}, l_{1} \mid n_{1}\right) \beta_{g_{2}}\left(k_{2},, l_{2} \mid n_{2}\right)\right) \alpha_{g_{1}}\left(k_{3}, n_{3}\right) \\
& \times\left.\left.\left. G^{\prime \prime}\left(\partial^{Q} H\left(F_{\lambda}(x, y)\right)\right)\right|_{x, y=\sqrt{3}} G^{\prime \prime}\left(\partial^{R} H\left(F_{\lambda}(x, y)\right)\right)\right|_{x, y=\sqrt{3}} G^{\prime}\left(\partial^{S} H\left(F_{\lambda}(x, y)\right)\right)\right|_{x, y=2 \sqrt{3}} \\
& \times\left(\left.\left.\left.\partial_{F_{\lambda}}^{k_{1}} H\left(F_{\lambda}(x, y)\right)\right|_{x, y=\sqrt{3}} \partial_{F_{\lambda}}^{l_{1}} H\left(F_{\lambda}(x, y)\right)\right|_{x, y=\sqrt{3}} \partial_{F_{\lambda}}^{k_{2}} H\left(F_{\lambda}(x, y)\right)\right|_{x, y=\sqrt{3}}\right. \\
& \left.\times\left.\partial_{F_{\lambda}}^{l_{2}} H\left(F_{\lambda}(x, y)\right)\right|_{x, y=\sqrt{3}}\right)\left.\partial_{F_{\lambda}}^{k_{3}} H\left(F_{\lambda}(x, y)\right)\right|_{x, y=2 \sqrt{3}}
\end{aligned}
$$

$K_{4}\left(G\left(H\left(F_{\lambda}\right)\right)\right)$

$$
=\sum_{n_{1}=0}^{\infty} \sum_{n_{2}=0}^{\infty} \sum_{n_{3}=0}^{\infty} \sum_{k_{1}, l_{1}=0 ; k_{1}<l_{1}}^{k_{1}+l_{1}=n_{1}} \sum_{k_{2}, l_{2}=0 ; k_{2}<l_{2}}^{k_{2}+l_{2}=n_{2}} \sum_{k_{3}=0 ; k_{3}<l_{3}}^{n_{3}} \sum_{Q=1}^{k_{1}+l_{1}-1} \sum_{R=1}^{k_{2}+l_{2}-1} \sum_{S=1}^{k_{3}+l_{3}-1}
$$

$$
\left(\beta_{g_{1}}\left(k_{1}, l_{1} \mid n_{1}\right) \beta_{g_{2}}\left(k_{2}, l_{2} \mid n_{2}\right) \beta_{g_{3}}\left(k_{3}, l_{3} \mid n_{3}\right)+\beta_{g_{1}}\left(k_{1}, l_{1} \mid n_{1}\right) \beta_{g_{3}}\left(k_{2}, l_{2} \mid n_{2}\right) \beta_{g_{2}}\left(k_{3}, l_{3} \mid n_{3}\right)\right.
$$$$
+\beta_{g_{1}}\left(k_{2}, l_{2} \mid n_{2}\right) \beta_{g_{2}}\left(k_{1}, l_{1} \mid n_{1}\right) \beta_{g_{3}}\left(k_{3}, l_{3} \mid n_{3}\right)+\beta_{g_{1}}\left(k_{2}, l_{2} \mid n_{2}\right) \beta_{g_{3}}\left(k_{1}, l_{1} \mid n_{1}\right) \beta_{g_{2}}\left(k_{3}, l_{3} \mid n_{3}\right)
$$$$
\left.+\beta_{g_{1}}\left(k_{3}, l_{3} \mid n_{3}\right) \beta_{g_{2}}\left(k_{1}, l_{1} \mid n_{1}\right) \beta_{g_{3}}\left(k_{2}, l_{2} \mid n_{2}\right)+\beta_{g_{1}}\left(k_{3}, l_{3} \mid n_{3}\right) \beta_{g_{3}}\left(k_{1}, l_{1} \mid n_{1}\right) \beta_{g_{2}}\left(k_{2}, l_{2} \mid n_{2}\right)\right)
$$$$
\times\left.\left.\left. G^{\prime \prime}\left(\partial^{Q} H\left(F_{\lambda}(x, y)\right)\right)\right|_{x, y=\sqrt{3}} G^{\prime \prime}\left(\partial^{R} H\left(F_{\lambda}(x, y)\right)\right)\right|_{x, y=\sqrt{3}} G^{\prime \prime}\left(\partial^{S} H\left(F_{\lambda}(x, y)\right)\right)\right|_{x, y=2 \sqrt{3}}
$$$$
\times\left(\left.\left.\left.\partial_{F_{\lambda}}^{k_{1}} H\left(F_{\lambda}(x, y)\right)\right|_{x, y=\sqrt{3}} \partial_{F_{\lambda}}^{l_{1}} H\left(F_{\lambda}(x, y)\right)\right|_{x, y=\sqrt{3}} \partial_{F_{\lambda}}^{k_{2}} H\left(F_{\lambda}(x, y)\right)\right|_{x, y=\sqrt{3}}\right.
$$$$
\left.\times\left.\left.\left.\partial_{F_{\lambda}}^{l_{2}} H\left(F_{\lambda}(x, y)\right)\right|_{x, y=\sqrt{3}} \partial_{F_{\lambda}}^{k_{3}} H\left(F_{\lambda}(x, y)\right)\right|_{x, y=2 \sqrt{3}} \partial_{F_{\lambda}}^{k_{3}} H\left(F_{\lambda}(x, y)\right)\right|_{x, y=2 \sqrt{3}}\right)
$$

where, in our notations, $G\left(\partial^{Q} H\right)$ is obtained from $G(H)$ by replacing the argument $H \rightarrow$ $\partial^{Q} H$ and $\partial^{Q} H \equiv \frac{\partial^{Q} H}{\partial F_{\lambda}}$ and finally $G^{\prime}\left(\partial^{Q} H\right)=\frac{\partial G}{\partial\left(\partial^{Q} H\right)}$. This concludes the computation of the three-point SFT correlator for our solution ansatz. The final step is to compute the kinetic term $<<Q \Psi(0) \mid I \circ \Psi(0)>>$ using the operator products and the identities derived 
above. According to the BRST transformation identity (94) this correlator is determined by two contributions: one proportional to the ghost part $<c(z) \partial c c\left(w>\left.\right|_{z=0 ; w \rightarrow \infty}=\right.$ $(z-w)^{2}$ another to $\left\langle c(z) \partial^{2} c c(w)>\left.\right|_{z=0 ; w \rightarrow \infty}=-2(z-w)\right.$. Note that, since $c, \partial c c$ and $\partial^{2} c c$ ghost fields have conformal dimensions $-1,-1$ and 0 respectively, and since the conformal transformation by $I(z)$ takes 0 to infinity, it is straightforward to check that the matter part of the first contribution only contains the terms with the conformal dimensions of the string field components at $z$ equal to those of the string components at $w$; all the terms with unequal conformal dimensions of operators at $z$ and $w$ vanish in the limit $w \rightarrow \infty$. Similarly, the matter part of the second contribution (multiplied by the $<c(z) \partial^{2} c c(w)>\left.\right|_{z=0 ; w \rightarrow \infty}$ ghost correlator) only contains the terms with the conformal dimensions of the operators at $z$ equal to those of the operators at $w$ plus one.

Then, performing straightforward calculation of the correlator, similar to those above, plugging into SFT equations of motion (1) leads to the defining relation for the $G\left(H\left(F_{\lambda}\right)\right)$ function of our ansatz, given by:

$$
\begin{array}{r}
\sum_{n=0}^{\infty} \sum_{k=1}^{n} \alpha_{I}(k, n) \sum_{Q=0}^{k}\left\{Z_{0}\left[\tilde{G}\left(H\left(F_{\lambda}\right)\right) \mid G^{\prime}\left(\partial_{F_{\lambda}}^{Q} H\left(F_{\lambda}\right)\right)\right]\right. \\
\left.\left.-Z_{1}\left[\partial_{F_{\lambda}} \tilde{G}\left(H\left(F_{\lambda}\right)\right) \mid G^{\prime}\left(\partial_{F_{\lambda}}^{Q} \tilde{H}\left(F_{\lambda}\right)\right)\right]\right\}\right]\left.\partial_{F_{\lambda}}^{k} \tilde{H}\left(F_{\lambda}\right)\right|_{x=y=1} \\
\left.+\sum_{n=0}^{\infty} \sum_{k, l=1 ; k<l}^{n} \beta_{I}(k, l \mid n) \sum_{Q=0}^{k+l-1}\right\} Z_{0}\left[\tilde{G}\left(\tilde{H}\left(F_{\lambda}\right)\right) \mid G^{\prime \prime}\left(\partial_{F_{\lambda}}^{Q} \tilde{H}\left(F_{\lambda}\right)\right)\right] \\
\left.\left.-Z_{1}\left[\partial_{F_{\lambda}} \tilde{G}\left(H\left(F_{\lambda}\right)\right) \mid G^{\prime \prime}\left(\partial_{F_{\lambda}}^{Q} \tilde{H}\left(F_{\lambda}\right)\right)\right]\right\} \partial_{F_{\lambda}}^{k} \tilde{H}\left(F_{\lambda}\right)\left|\partial_{F_{\lambda}}^{l} \tilde{H}\left(F_{\lambda}\right)\right|_{x, y=1}\right\} \\
=\sum_{j} K_{j}\left(G\left(H\left(F_{\lambda}\right)\right)\right)
\end{array}
$$

where the operations $Z_{k}\left[f_{1}(x) \mid f_{2}(x)\right]$ acting on functions $f_{1}$ and $f_{2}$ are defined as follows: if $f_{1}(x)=\sum_{m} a_{m} x^{m}$ and $f_{2}(x)=\sum_{n} a_{n} x^{n}$ are the series expansions for $f_{1}$ and $f_{2}$ then $Z_{k}$ maps them into the function (formal series)

$$
Z_{k}\left[f_{1}(x) \mid f_{2}(y)\right]=\sum_{n} a_{n} b_{n+k} x^{n} y^{n+k}
$$

The tilde operations are again defined according to:

$$
\begin{array}{r}
\tilde{G}(H(x))=x \frac{d}{d x} G(H(x))-G(H(x)) \\
\tilde{H}(x)=x \frac{d}{d x} H(x)-H(x)
\end{array}
$$


and the $K_{j}$-functions are defined by (101)-(104). The functional equation (105) is the main result of this paper and constitutes the defining relation for the SFT ansatz. As cumbersome as this relation is, it can be, e.g., solved order by order by iterations and reduces the SFT equation (1) to the identity which is essentially algebraic. In the case of $\alpha^{2}=-2$ that we mostly have explored in this paper, one particularly simple example for the generating functions solving the defining relation (105) is given by

$$
\begin{array}{r}
G(H)=\frac{1}{1-H\left(F_{\lambda}\right)} \\
H(x)=\frac{1}{1-x}
\end{array}
$$

Replacing $x^{n} \rightarrow B_{\vec{\psi}}^{(n)}$ according to our usual prescription, leads to the generating function of the $\mathrm{SU}(2)$ enveloping algebra $T(\mu)$ with the parameter $\mu$ defined by the $F_{\lambda}$ (elementary correlator of Bell polynomial operators). The classical $w_{\infty}$ algebra is then recovered in the simple pole $\sim(z-w)^{-1}$ of the OPE of $G\left(H\left(B^{(n)}(z)\right)\right)$ and $G\left(H\left(B^{(n)}(w)\right)\right)$. In general, the defining relation (105) appears to parametrize the class of SFT solutions, of which (108) is an elementary example. Finding the explicit form of this class of the solutions , generalizing (108) appears to be an important challenge and obviously doesn't seem to be easy. However, it appears that this class is most naturally expressible in terms of the series in the powers of the generating function (108) for the products of the Bell polynomial operators, relating it to the enveloping of the enveloping of $S U(2)$ (and more particularly, to the enveloping of $w_{\infty}$ ) The objects like these are known to be relevant to the quantization of higher spin theories and to the multi-particle realizations of the higher-spin algebras [36]. The crucial point about the SFT solutions, constrained to the subspace of operators given by products of the Bell polynomials, is that these objects

a) behave in a controllable and consistent way in the SFT star product computations

b)form a natural operator basis for the free-field realization of the $S U(2)$ envelopings and $w_{\infty}$. In the concluding section, we shall briefly discuss how the construction, studied in this paper, can be generalized to higher space-time dimensions.

\section{Conclusion and Discussion}

In this paper we have considered the ansatz solution in bosonic string field theory, given by formal series in partial (incomplete) Bell polynomials of Bell polynomial operators in the worldsheet derivatives of the target space fields. These objects form an operator algebra realization for the enveloping of $S U(2)$, including the $w_{\infty}$ algebra appearing at the 
simple pole of the OPE. This, up to ideal factorization, is isomorphic to a chiral copy of higher spin algebra in $A d S_{3}$. The solution is given in terms of of the functional constraints on the generating functions for the operators realizing this enveloping. These constraints altogether are quite cumbersome and finding their manifest solutions doesn't appear to be an easy challenge, except for a relatively simple example (108).

Nevertheless, the constraints for the $h$ and $g$-expansion coefficients are essentially algebraic and in principle be analyzed order by order by iterations.

An important question is whether the construction, considered in our work, can be extended to SFT solutions involving higher-dimensional enveloping/higher spin algebras. A possible answer to that may come from superstring generalization of the computation performed in this work and switching on the $\beta-\gamma$ system of the superconformal ghosts. Just as the solution, considered in this paper, was in a sense inspired by the bosonic $c=1$ model (an elementary example pointing out the relevance of operator algebra involving Bell polynomial products to $w_{\infty}$ and higher spin algebra) one can use a supersymmetric $c=1$ model coupled to the $\beta-\gamma$ system as a toy model inspiration. It is known that the interaction with the superconformal ghosts enhances the $S U(2)$ symmetry at the selfdual point to $S U(N)$ where $N-2$ is the maximal superconformal ghost number (ghost cohomology rank) of the generators [37] One can hope that manifest form of the vertex operators in this model would prompt us the form of the ansatz we should be looking for, and the resulting solution would be relevant to envelopings of $S U(N)$ or their subalgebras, related to isometries of $A d S$ in different space-time dimensions. The manifest form of the vertex operators in this model would again involve the products of Bell polynomials, however their structure will be far more diverse. In the present paper the $\psi=\vec{\alpha} \vec{X}$ parameter of the operators was fixed to be the same for the all the string field components (as it is the same for all the operators for the bosonic discrete states and is equal to $-i \sqrt{2} X)$. Switching on the higher superconformal ghost pictures in the $c=1$ model would then result in the appearance of Bell polynomial products with mixed $\psi$-parameters. While the naive number of the parameters would be $\frac{1}{2} N(N-1)$ (total number of the lowering operators of $S U(N)$ ), the actual number would be less and of the order of $N$, since not all the lowering operators, acting on tachyonic primaries, lead to physically distinct states. The distinct states are basically generated by the lowering operators of ghost numbers $N-2$ carrying the maximum momentum value in the $X$-direction, equal to $N-1$, and the total number of such generators is $N-1$. Thus one can hope that introducing extra $\psi$-parameters will direct us towards the SFT solutions describing the higher-dimensional enveloping/ higher spin 
algebras. It looks plausible that the framework involving the WZW-type Berkovits string field theory may turn out to be a convenient framework for this program along with cubic superstring field theory with picture-changing insertions [2], [38], [21], [19]. Following this strategy, one can hope to find the defining constraints for the generating functions, similar to those considered in this paper. It would certainly be of interest and of importance to study these constraints and to identify some of their manifest solutions. This hopefully shall lead to new important insights regarding nonperturbative higher spin configurations, as well as to deeper understanding of the underlying relations between SFT and higher spin field theories, which appear to be crucial ingredients of holography principle in general.

\section{Acknowledgements}

It is a great pleasure to thank the speakers and the participants of the annual string field theory conference (SFT-2015) in Chengdu (May 11-16, 2015), as well as the speakers at the introductory school, for the illuminating lectures, talks and productive discussions. It is also a great pleasure to thank Bo Ning, Zheng Sun and Haitang Yang for our collaboration in organizing SFT-2015. 


\section{References}

[1] E. Witten, Nucl.Phys. B268 (1986) 253

[2] E. Witten, Phys.Rev. D46 (1992) 5467-5473

[3] M. A. Vasiliev, Phys. Lett. B 285 (1992) 225

[4] V. E. Lopatin and M. A. Vasiliev, Mod. Phys. Lett. A 3 (1988) 257

[5] E.S. Fradkin, M.A. Vasiliev, Phys. Lett. B 189 (1987) 89

[6] X. Bekaert, S. Cnockaert, C. Iazeolla, M. A. Vasiliev, hep-th/0503128

[7] M. Schnabl, Adv.Theor.Math.Phys. 10 (2006) 433-501

[8] T. Erler, M. Schnabl,JHEP 0910 (2009) 066

[9] M. Kroyter, Y. Okawa, M. Schnabl, S. Torii, B. Zwiebach, JHEP 1203 (2012) 030

[10] T. Erler, JHEP 1311 (2013) 007

[11] T. Erler, JHEP 1104 (2011) 107

[12] T. Erler, M. Schnabl, JHEP 0910 (2009) 066

[13] A. Sen, JHEP 9912 (1999) 027

[14] A. Sen, B. Zwiebach, JHEP 0003 (2000) 002

[15] N. Berkovits, A. Sen, B. Zwiebach, Nucl.Phys. B587 (2000) 147-178

[16] L. Bonora, C. Maccaferri, D.D. Tolla, JHEP 1111 (2011) 107

[17] C. Maccaferri, JHEP 1405 (2014) 004

[18] T. Erler, C. Maccaferri, JHEP 1410 (2014) 029

[19] C. Preitschopf, C. Thorn and S. Yost, Nucl. Phys. B337 (1990) 363

[20] I. Arefeva, P. Medvedev, A. Zubarev, Nucl.Phys. B341 (1990) 464-498

[21] I. Arefeva, A. Zubarev, Mod.Phys.Lett. A8 (1993) 1469-1476

[22] E.S. Fradkin and M.A. Vasiliev, Int. J. Mod. Phys. A 3 (1988) 2983

[23] N. Berkovits, Nucl. Phys. B450 (1995) 90

[24] N. Berkovits, JHEP 0004 (2000) 022

[25] L. Rastelli, B. Zwiebach, JHEP 0109 (2001) 038

[26] L. Rastelli, B. Zwiebach, JHEP 0109 (2001) 038

[27] L. Bonora, C. Maccaferri, D. Mamone, M. Salizzone, hep-th/0304270

[28] M. Douglas, H. Liu, G. Moore, B. Zwiebach, JHEP 0204 (2002) 022

[29] I. Bars, Y. Matsuo, Phys.Rev. D66 (2002) 066003

[30] I. Bars, I. Kishimoto, Y. Matsuo, Phys.Rev. D67 (2003) 066002

[31] I. Bars, hep-th/0211238

[32] I. Klebanov, A. Polyakov, Mod.Phys.Lett. A6 (1991) 3273-3281

[33] E. Witten, Nucl.Phys. B373 (1992) 187-213

[34] M. Henneaux, S.-J. Rey,JHEP 1012 (2010) 007

[35] C. Pope, L. Romans, X. Shen, Nucl. Phys. B339 (1990) 191

[36] M. Vasiliev, Class.Quant.Grav. 30 (2013) 104006

[37] D. Polyakov, Phys.Rev. D90 (2014) 8, 086002

[38] I. Arefeva, P. Medvedev, A. Zubarev, Mod. Phys. Lett. A6, 949 (1991) 